\title{
ON A SPLITTING SCHEME FOR THE NONLINEAR SCHRÖDINGER EQUATION IN A RANDOM MEDIUM*
}

\author{
RENAUD MARTY ${ }^{\dagger}$
}

\begin{abstract}
In this paper we consider a nonlinear Schrödinger equation (NLS) with random coefficients, in a regime of separation of scales corresponding to diffusion approximation. The primary goal of this paper is to propose and study an efficient numerical scheme in this framework. We use a pseudo-spectral splitting scheme and we establish the order of the global error. In particular we show that we can take an integration step larger than the smallest scale of the problem, here the correlation length of the random medium. We study the asymptotic behavior of the numerical solution in the diffusion approximation regime.
\end{abstract}

Key words. Light waves, random media, asymptotic theory, splitting scheme.

AMS subject classifications. 35Q55, 60F05, 65M12

\section{Introduction}

Optical fibers have been extensively studied because they play an important role in modern communication systems $[1,2]$. In particular, the limitation effects of high bit rate transmission and numerical simulations of pulse dynamics have attracted attention of engineers, physicists an applied mathematicians.

One of the main limitations of high bit transmission in optical fiber is the chromatic dispersion. The different frequency components of the pulse have different phase velocities, which gives pulse spreading. Solutions have been proposed to compensate for the pulse broadening induced by dispersion. A widely used method consists of the periodic compensation of accumulated dispersion by insertion of an additional piece of fiber with a well controlled length and dispersion values. So, we obtain a fiber with dispersion coefficient which is random and fluctuating around its zero mean value. We shall consider such a fiber in this paper.

The evolution of the electric field in an optical fiber with constant dispersion coefficient is governed by the nonlinear Schrödinger equation (NLS)

$$
i \frac{\partial u}{\partial z}=d_{0} \frac{\partial^{2} u}{\partial t^{2}}+n_{0}|u|^{2} u
$$

where $d_{0}$ (resp. $n_{0}$ ) is the dispersion (resp. nonlinearity) coefficient. A lot of work has been devoted to the numerical study of this equation using many different discretization schemes. In particular, some papers are devoted to the study of order estimates for splitting methods $[5,9,14]$. Splitting methods are based on the decomposition of the flow of (1.1) into the flows of two partial problems: the linear Schrödinger equation (with $n_{0}=0$ ) and the nonlinear equation (1.1) with $d_{0}=0$. These partial problems can be solved explicitly.

In this paper we consider the pulse propagation in an optical fiber with a zeromean random dispersion coefficient. The electric field is a solution of a nonlinear Schrödinger equation with random coefficients. One of our goals is to study numerically the evolution of the electric field. We use a Fourier split-step method. This

\footnotetext{
${ }^{*}$ Received: March 17, 2006; accepted (in revised version): July 31, 2006. Communicated by Lenya Ryzhik.

†Laboratoire de Statistique et Probabilités, Université Paul Sabatier, 118 Route de Narbonne, 31062 Toulouse, France (marty@cict.fr). New address: Department of Mathematics, University of California at Irvine, Irvine, CA 92697-3875, USA (rmarty@math.uci.edu).
} 
method has already been applied to homogeneous equations, but also to random equations $[3,4]$.

Different scales are present in the problem: the fiber length, the correlation length of the medium, the typical dispersion length and the nonlinearity length. We take into account these different scales to study the asymptotics of the field. We obtain a limit theorem for the solution of the random nonlinear Schrödinger equation. This limit can be identified as the solution of a stochastic NLS-type equation. We can cite other works dealing with different forms of stochastic NLS equations $[7,8]$. We propose two proofs for our limit theorem. The first one is based on the continuity of the Itô's map of the propagation equation. The second one is based on the error estimate for the numerical solution and approximation-diffusion theorems.

Section 2 is devoted to the presentation of the pulse propagation model in a randomly perturbed optical fiber. In Section 3 we study the nonlinear Schrödinger equation driven by an arbitrary deterministic continuous noise. In Section 4 we propose a numerical scheme and we establish an error estimate. In Section 5 we study the asymptotic behavior of the electric field. Section 6 is devoted to extend previous results to a more realistic nonlinear model for a pulse propagation. In Section 7 we give a formal approach for the global error computation to improve the results of Section 4. Section 8 is devoted to numerical simulations.

\section{Notations.}

- $L^{2}$ denotes the space of functions $L^{2}(\mathbb{R}, \mathbb{C})$. For every $p \in \mathbb{N}, H^{p}$ denotes the Sobolev space $H^{p}(\mathbb{R}, \mathbb{C})$.

- The Fourier transform of a function $\psi \in L^{2}$ is denoted by $\mathcal{F}(\psi)$ or by $\widehat{\psi}$.

\section{Formulation}

The electric field evolution in an optical fiber with a zero-mean dispersion coefficient is governed by the random nonlinear Schrödinger equation [1]:

$$
i \frac{\partial u}{\partial z}+\varepsilon m(z) \frac{\partial^{2} u}{\partial t^{2}}+\varepsilon^{2} f\left(|u|^{2}\right) u=0, \quad u(z=0, t)=u_{0}(t) .
$$

$m$ is a centered stationary process and models the dispersion coefficient. $f$ is an increasing function which models the nonlinear response of the medium to the electric field. The initial condition $u_{0}$ is assumed to be in the Sobolev space $H^{2}$.

We have written Equation (2.1) in the microscopic scale where the correlation length and the initial pulse width are of order $1 . m$ models the fluctuations of the dispersion coefficient. In the microscopic scale the amplitude of the dispersion is small. So we introduce a small dimensionless parameter $\varepsilon \ll 1$ so that the dispersion term is written as $\varepsilon m(z) \partial^{2} u / \partial t^{2}$. Hence, in the linear approximation, the propagation can be modeled by the linear Schrödinger equation

$$
i \frac{\partial u}{\partial z}+\varepsilon m(z) \frac{\partial^{2} u}{\partial t^{2}}=0, \quad u(z=0, t)=u_{0}(t),
$$

and the pulse amplitude is of order 1 . We would like to study the role of the nonlinearity and its interplay with the dispersion term. We will see that the nonlinear effects are of order 1 if the nonlinearity parameter is rescaled by $\varepsilon^{2}$. So we consider Equation (2.1).

We consider the propagation equation in the macroscopic scale. We introduce the rescaled field $u^{\varepsilon}(z, t)=u\left(z / \varepsilon^{2}, t\right)$ which solves

$$
i \frac{\partial u^{\varepsilon}}{\partial z}+\frac{1}{\varepsilon} m\left(\frac{z}{\varepsilon^{2}}\right) \frac{\partial^{2} u^{\varepsilon}}{\partial t^{2}}+f\left(\left|u^{\varepsilon}\right|^{2}\right) u^{\varepsilon}=0, \quad u(z=0, t)=u_{0}(t) .
$$


We now give more precise conditions on the dispersion process $m$ and the nonlinearity function $f . m$ is a Markov process with infinitesimal generator $\mathcal{L}$. We assume that $m$ is centered, stationary, with values in a compact space, and satisfying Doeblin's condition $[12,13]$. As a consequence, $m$ admits a unique invariant probability measure, and its infinitesimal generator satisfy the Fredholm alternative. For instance, we can think of the process $m$ as a continuous Markov process with values in a compact interval, or a càd-làg Markov process with values in a finite set.

We assume that the nonlinearity function $f$ is of class $\mathcal{C}^{\infty}\left(\mathbb{R}_{+}, \mathbb{R}_{+}\right)$. We further assume that there exists a constant $M>0$ such that $f(x)=x$ if $x \leq M, f(x)=3 M / 2$ if $x \geq 2 M,\left\|f^{\prime}\right\|_{\infty} \leq 1$ and $\left\|f^{\prime \prime}\right\|_{\infty} \leq 1$. So, the function $g: \zeta \mapsto f\left(|\zeta|^{2}\right) \zeta$ and all its partial derivatives $^{1}$ are Lipschitz functions. The case where $f(x)=x$ for all $x \geq 0$, which corresponds to the standard cubic NLS equation, will be considered in Section 6 .

The first goal of this paper is to study Equation (2.2) numerically. Then we establish an effective propagation equation for electric field when $\varepsilon \rightarrow 0$ for $u^{\varepsilon}$.

\section{Nonlinear Schrödinger equation driven by a continuous noise}

In this section we study nonlinear Schrödinger equation driven by a continuous noise $w$ :

$$
u(z, t)=u_{0}(t)+i \sigma_{0} \int_{z_{0}}^{z} \frac{\partial^{2} u}{\partial t^{2}}\left(z^{\prime}, t\right) d w\left(z^{\prime}\right)+i \int_{z_{0}}^{z} f\left(\left|u\left(z^{\prime}, t\right)\right|^{2}\right) u\left(z^{\prime}, t\right) d z^{\prime},
$$

with $\sigma_{0} \in \mathbb{R}, z_{0}, z \in[0,1]\left(z_{0} \leq z\right)$ and $u_{0} \in L^{2}$. The goal of this section is to show that the solution is unique in $\mathcal{C}\left(\left[z_{0}, 1\right], L^{2}\right)$ and depends continuously on $w$.

3.1. Existence and uniqueness of the solution. The goal of this subsection is to give a sense to the solution for (3.1). The problem is more suitably written in the mild form as:

$$
u(z, t)=X_{w}\left(z_{0}, z\right) u_{0}(t)+i \int_{z_{0}}^{z} X_{w}\left(z^{\prime}, z\right) f\left(\left|u\left(z^{\prime}, t\right)\right|^{2}\right) u\left(z^{\prime}, t\right) d z^{\prime},
$$

where the parameter-dependent inhomogeneous operator $X_{w}$ is defined as follows

For a function $v_{0} \in L^{2}$, we give a sense to the solution for the linear problem:

$$
v(z, t)=v_{0}(t)+i \sigma_{0} \int_{z_{0}}^{z} \frac{\partial^{2} v}{\partial t^{2}}\left(z^{\prime}, t\right) d w\left(z^{\prime}\right) .
$$

We first assume that $w \in \mathcal{C}^{1}([0,1], \mathbb{R})$. We replace in $(3.3)$ the differential term $d w\left(z^{\prime}\right)$ by $w^{\prime}\left(z^{\prime}\right) d z^{\prime}$ where $w^{\prime}$ is the derivative of $w$. The unique solution of (3.3) in this case is

$$
v(z, t)=\frac{1}{\sqrt{4 i \pi \sigma_{0} w\left(z_{0}, z\right)}} \int_{-\infty}^{\infty} \exp \left(\frac{i\left(t-t^{\prime}\right)^{2}}{4 \sigma_{0} w\left(z_{0}, z\right)}\right) v_{0}\left(t^{\prime}\right) d t^{\prime}
$$

with

$$
w\left(z_{0}, z\right)=\int_{z_{0}}^{z} w^{\prime}\left(z^{\prime}\right) d z^{\prime}=w(z)-w\left(z_{0}\right)
$$

We note that the expression of the solution $v(z, t)$ depends only on the increments of $w$. So, we use the formulation of this solution to define the solution of (3.3) for an

\footnotetext{
${ }^{1}$ In this paper $g^{\prime}$ (resp. $g^{\prime \prime}$ ) denotes the gradient vector (resp. Hessian matrix) of $g$.
} 
arbitrary continuous noise $w$. We denote by $X_{w}\left(z_{0}, z\right)$ the flow associated to (3.3) for an initial condition at $z=z_{0}$ :

$$
X_{w}\left(z_{0}, z\right) v_{0}(t)=\frac{1}{\sqrt{4 i \pi \sigma_{0} w\left(z_{0}, z\right)}} \int_{-\infty}^{\infty} \exp \left(\frac{i\left(t-t^{\prime}\right)^{2}}{4 \sigma_{0} w\left(z_{0}, z\right)}\right) v_{0}\left(t^{\prime}\right) d t^{\prime}
$$

with $w\left(z_{0}, z\right)=w(z)-w\left(z_{0}\right)$. We can write $X_{w}\left(z_{0}, z\right) v_{0}$ in the Fourier domain as

$$
\mathcal{F}\left(X_{w}\left(z_{0}, z\right) v_{0}\right)(\theta)=\mathcal{F}\left(v_{0}\right)(\theta) \exp \left(-i \theta^{2} \sigma_{0} w\left(z_{0}, z\right)\right)
$$

So, the flow $X_{w}\left(z_{0}, z\right): L^{2} \rightarrow L^{2}$ is well-defined. Moreover, by Parseval's theorem, we have for every $p \in \mathbb{N},\left\|X_{w}\left(z_{0}, z\right) v_{0}\right\|_{H^{p}}=\left\|v_{0}\right\|_{H^{p}}$, that is to say that $X_{w}\left(z_{0}, z\right)$ is unitary in every Sobolev space $H^{p}$. We denote by $X\left(z_{0}, z\right)$ the flow $X_{w}\left(z_{0}, z\right)$ if there is no ambiguity on the noise $w$.

Now we can establish the existence and the uniqueness of the solution of (3.2).

THEOREM 3.1. Let $u_{0} \in L^{2}$. Equation (3.2) admits a unique solution $u$ in $\mathcal{C}\left(\left[z_{0}, 1\right], L^{2}\right)$. stated.

The proof of Theorem 3.1 will be delayed until several technical lemmas can be

We denote by $S_{w}\left(z_{0}, z\right)$ (or $S\left(z_{0}, z\right)$ if there is not ambiguity on the noise $w$ ) the flow which is associated to (3.2) with initial condition in $z=z_{0}$.

Without loss of generality we assume $z_{0}=0$. To prove Theorem 3.1 we first consider the sequence of functions $\left((z, t) \mapsto x_{n}(z, t)\right)_{n \in \mathbb{N}}$ which is defined by: $x_{0}(z, t)=$ $u_{0}(t)$ and for every $n>0$ :

$$
x_{n}(z, t)=X(0, z) u_{0}(t)+i \int_{0}^{z} X\left(z^{\prime}, z\right) f\left(\left|x_{n-1}\left(z^{\prime}, t\right)\right|^{2}\right) x_{n-1}\left(z^{\prime}, t\right) d z^{\prime} .
$$

If $f$ is bounded, then $t \rightarrow f\left(\left|x_{n-1}\left(z^{\prime}, t\right)\right|^{2}\right) x_{n-1}\left(z^{\prime}, t\right)$ belongs to $L^{2}$ for all $z$ and $n$. So $\left(x_{n}\right)_{n}$ is a well-defined sequence of functions with integrable squared modulus with respect to $t$.

We establish a regularity result for $x_{n}$ in Lemma 3.3 which is a consequence of the following technical lemma.

LEMMA 3.2. (i) Let $\left(\phi_{n}\right)_{n}$ be a sequence of measurable and nonnegative functions. If there exist two positive constants $C_{1}$ and $C_{2}$ such that $\phi_{0} \equiv C_{1}$ and for all $n \in \mathbb{N}^{*}$ and $z \in[0,1]$

$$
\phi_{n+1}(z) \leq C_{1}+C_{2} \int_{0}^{z} \phi_{n}\left(z^{\prime}\right) d z^{\prime}
$$

then

$$
\sup _{z \in[0,1]} \phi_{n}(z) \leq C_{1} \exp \left(C_{2}\right)
$$

(ii) Let $\left(\phi_{n}\right)_{n}$ be a sequence of measurable and nonnegative functions. If there exist two positive constants $C_{3}$ and $C_{4}$ such that for all $n \in \mathbb{N}^{*}$ and $z \in[0,1], \phi_{0}(z) \leq C_{4}$ and

$$
\phi_{n+1}(z) \leq C_{3} \int_{0}^{z} \phi_{n}\left(z^{\prime}\right) d z^{\prime}
$$


then

$$
\sup _{z \in[0,1]} \phi_{n}(z) \leq C_{4} \frac{C_{3}^{n}}{n !}
$$

Proof. For (i) we prove by recurrence with respect to $n$ that $\phi_{n}(z) \leq C_{1} \exp \left(C_{2} z\right)$. For (ii) we prove by recurrence with respect to $n$ that $\phi_{n}(z) \leq C_{4}\left(C_{3} z\right)^{n} / n$ ! .

LEMmA 3.3. If $u_{0} \in H^{2}$, then the sequence $\left(x_{n}\right)_{n}$ is bounded in $H^{p}(\mathbb{R}, \mathbb{C})$ for all $p=0,1,2$, uniformly with respect to $z \in[0,1]$ and $n \in \mathbb{N}$, that is to say that there exists a constant $C$ which depends only on $f$ and $\left\|u_{0}\right\|_{H^{2}}$ such that for all $n \in \mathbb{N}$ we have

$$
\sup _{z \in[0,1]}\left\|x_{n}(z, \cdot)\right\|_{H^{2}} \leq C .
$$

Proof. Let $p=0 . X\left(z_{0}, z\right)$ is unitary, then

$$
\left\|x_{n+1}(z, \cdot)\right\|_{L^{2}} \leq\left\|u_{0}\right\|_{L^{2}}+\|f\|_{\infty} \int_{0}^{z}\left\|x_{n}\left(z^{\prime}, \cdot\right)\right\|_{L^{2}} d z^{\prime} .
$$

By Lemma 3.2, we get

$$
\sup _{z \in[0,1]}\left\|x_{n}(z, \cdot)\right\|_{L^{2}} \leq\left\|u_{0}\right\|_{L^{2}} \exp \left(\|f\|_{\infty}\right) .
$$

Let $p=1$. From (3.4), if $x_{n} \in H^{1}$ then $x_{n+1} \in H^{1}$, and $X\left(z_{0}, z\right)$ is unitary, then

$$
\left\|\partial_{t} x_{n+1}(z, \cdot)\right\|_{L^{2}} \leq\left\|\partial_{t} u_{0}\right\|_{L^{2}}+\left\|g^{\prime}\right\|_{\infty} \int_{0}^{z}\left\|\partial_{t} x_{n}\left(z^{\prime}, \cdot\right)\right\|_{L^{2}} d z^{\prime} .
$$

By Lemma 3.2 we have

$$
\sup _{z \in[0,1]}\left\|x_{n}(z, \cdot)\right\|_{H^{1}} \leq\left\|u_{0}\right\|_{H^{1}} \exp \left(\|f\|_{\infty}+\left\|g^{\prime}\right\|_{\infty}\right) .
$$

Let $p=2 . X\left(z_{0}, z\right)$ is unitary, so

$$
\begin{aligned}
\left\|\partial_{t}^{2} x_{n+1}(z, \cdot)\right\|_{L^{2}} \leq & \left\|\partial_{t}^{2} u_{0}\right\|_{L^{2}} \\
& +\left\|g^{\prime}\right\|_{\infty} \int_{0}^{z}\left\|\partial_{t}^{2} x_{n}\left(z^{\prime}, \cdot\right)\right\|_{L^{2}} d z^{\prime} \\
& +\left\|g^{\prime \prime}\right\|_{\infty} \int_{0}^{z}\left\|\partial_{t} x_{n}\left(z^{\prime}, \cdot\right)\right\|_{L^{4}}^{2} d z^{\prime} .
\end{aligned}
$$

By Gagliardo-Nirenberg inequality

$$
\|\cdot\|_{L^{4}}^{2} \leq C_{g n}\|\cdot\|_{H^{1}}\|\cdot\|_{L^{2}},
$$

and the beginning of the proof, there exists $C$ which depends on $\left\|u_{0}\right\|_{H^{1}}$ and $f$ such that

$$
\left\|x_{n+1}(z, \cdot)\right\|_{H^{2}} \leq\left\|u_{0}\right\|_{H^{2}}+C \int_{0}^{z}\left\|x_{n}\left(z^{\prime}, \cdot\right)\right\|_{H^{2}} d z^{\prime} .
$$


Then, by lemma 3.2 and the previous estimates, we have

$$
\sup _{z \in[0,1]}\left\|x_{n}(z, \cdot)\right\|_{H^{2}} \leq\left\|u_{0}\right\|_{H^{2}} \exp (C) .
$$

This ends the proof.

Before proving Theorem 3.1 we establish the two following lemmas.

Lemma 3.4. If $u_{0} \in L^{2}$, then $\left(x_{n}\right)_{n}$ is a Cauchy sequence in $L^{\infty}\left([0,1], L^{2}\right)$.

Proof. Let $p$ and $q \in \mathbb{N}$. Since $g^{\prime}$ is bounded, from (3.4):

$$
\begin{aligned}
\left\|x_{p+q+1}(z)-x_{q+1}(z)\right\|_{L^{2}} & \leq \int_{0}^{z}\left\|g\left(x_{p+q}\left(z^{\prime}\right)\right)-g\left(x_{q}\left(z^{\prime}\right)\right)\right\|_{L^{2}} d z^{\prime} \\
& \leq\left\|g^{\prime}\right\|_{\infty}^{q+1} \int_{0}^{z} d z_{1} \int_{0}^{z_{1}} d z_{2} \ldots \int_{0}^{z_{q}}\left\|x_{p}\left(z_{q+1}\right)-x_{0}\right\|_{L^{2}} d z_{q+1} .
\end{aligned}
$$

From (3.5), we get

$$
\begin{aligned}
\left\|x_{p}\left(z_{q+1}\right)-u_{0}\right\|_{L^{2}} & \leq\left\|u_{0}\right\|_{L^{2}}+\left\|x_{p}\left(z_{q+1}\right)\right\|_{L^{2}} \\
& \leq\left\|u_{0}\right\|_{L^{2}}\left(1+\exp \left(\|f\|_{\infty}\right)\right) .
\end{aligned}
$$

Substituting into (3.6) and taking the supremum in $z \in[0,1]$, we obtain

$$
\sup _{z \in[0,1]}\left\|x_{p+q+1}(z)-x_{q+1}(z)\right\|_{L^{2}} \leq\left\|u_{0}\right\|_{L^{2}}\left(1+\exp \left(\|f\|_{\infty}\right)\right) \frac{\left\|g^{\prime}\right\| \|_{\infty}^{q+1}}{(q+1) !} .
$$

Letting $q \rightarrow \infty$, we establish that $\left(x_{n}\right)_{n}$ is a Cauchy sequence in $L^{\infty}\left([0,1], L^{2}\right)$.

LEMMA 3.5. The map

$$
\begin{aligned}
\mathcal{A}: L^{\infty}\left([0,1], L^{2}\right) & \rightarrow L^{\infty}\left([0,1], L^{2}\right) \\
v & \mapsto X(0, z) u_{0}(t)+i \int_{0}^{z} X\left(z^{\prime}, z\right) g\left(v\left(z^{\prime}, t\right)\right) d z^{\prime},
\end{aligned}
$$

is continuous.

Proof. Let $v_{1}$ and $v_{2}$ in $L^{\infty}\left([0,1], L^{2}\right)$. We have

$$
\begin{aligned}
\left\|\mathcal{A}\left(v_{1}\right)(z)-\mathcal{A}\left(v_{2}\right)(z)\right\|_{L^{2}} & \leq\left\|\int_{0}^{z} X\left(z^{\prime}, z\right) g\left(v_{1}\left(z^{\prime}\right)\right) d z^{\prime}-\int_{0}^{z} X\left(z^{\prime}, z\right) g\left(v_{2}\left(z^{\prime}\right)\right) d z^{\prime}\right\|_{L^{2}} \\
& \leq \int_{0}^{z}\left\|g\left(v_{1}\left(z^{\prime}\right)\right)-g\left(v_{2}\left(z^{\prime}\right)\right)\right\|_{L^{2}} d z^{\prime} \\
& \leq\left\|g^{\prime}\right\|_{\infty} \int_{0}^{z}\left\|v_{1}\left(z^{\prime}\right)-v_{2}\left(z^{\prime}\right)\right\|_{L^{2}} d z^{\prime}
\end{aligned}
$$

then

$$
\left\|\mathcal{A}\left(v_{1}\right)-\mathcal{A}\left(v_{2}\right)\right\|_{L^{\infty}\left([0,1], L^{2}\right)} \leq\left\|g^{\prime}\right\|_{\infty}\left\|v_{1}-v_{2}\right\|_{L^{\infty}\left([0,1], L^{2}\right)}
$$

The continuity of $\mathcal{A}$ follows.

Now we prove Theorem 3.1. 
Proof. (Theorem 3.1) $\left(x_{n}\right)_{n}$ is a Cauchy sequence in $L^{\infty}\left([0,1], L^{2}\right)$, so it converges to a limit $u$ in $L^{\infty}\left([0,1], L^{2}\right)$. By the continuity of $\mathcal{A}, u$ is solution to (3.2). Now we prove that $u \in \mathcal{C}\left([0,1], L^{2}\right) . X\left(z_{0}, z\right)$ is unitary in $L^{2}$, so for $z_{2}<z_{1}$ :

$$
\begin{aligned}
\left\|u\left(z_{1}\right)-u\left(z_{2}\right)\right\|_{L^{2}} \leq & \left\|\left(X\left(0, z_{1}\right)-X\left(0, z_{2}\right)\right) u_{0}\right\|_{L^{2}}+\int_{z_{2}}^{z_{1}}\left\|g\left(u\left(z^{\prime}\right)\right)\right\|_{L^{2}} d z^{\prime} \\
& +\int_{0}^{z_{2}}\left\|\left(X\left(z^{\prime}, z_{1}\right)-X\left(z^{\prime}, z_{2}\right)\right) g\left(u\left(z^{\prime}\right)\right)\right\|_{L^{2}} d z^{\prime} .
\end{aligned}
$$

An application of bounded convergence theorem gives $u \in \mathcal{C}\left([0,1], L^{2}\right)$. Hence, (3.2) has a solution in $\mathcal{C}\left([0,1], L^{2}\right)$.

It remains to prove the uniqueness of this solution. Let $\widetilde{u}$ be another solution. The facts that $X\left(z_{0}, z\right)$ is unitary and $g$ is Lipschitz give for every $z \in[0,1]$ :

$$
\|u(z)-\widetilde{u}(z)\|_{L^{2}} \leq C \int_{0}^{z}\left\|u\left(z^{\prime}\right)-\widetilde{u}\left(z^{\prime}\right)\right\|_{L^{2}} d z^{\prime} .
$$

So by Gronwall's lemma, for every $z \in[0,1]$ :

$$
\| u(z)-\left.\widetilde{u}(z)\right|_{L^{2}}=0,
$$

which implies uniqueness.

We prove a result on the regularity of the solution of (3.2).

Lemma 3.6. Let $u_{0} \in H^{2}$. For every $z \in[0,1], u(z, \cdot) \in H^{2}$. Moreover:

$$
\sup _{z \in[0,1]}\|u(z, \cdot)\|_{H^{2}}<\infty,
$$

and

$$
\sup _{z \in[0,1]}\|g(u(z, \cdot))\|_{H^{2}}<\infty .
$$

Proof. We fix $z \in[0,1]$. By Lemma 3.3, $\left(x_{n}(z)\right)_{n}$ is in a closed ball of $H^{2}$. Hence, we can extract a subsequence $\left(x_{n_{k}}(z)\right)_{k}$ which converges weakly in $H^{2}$ to a limit $y(z) \in H^{2}$ (because $H^{2}$ is reflexive) and, consequently, in $L^{2}$. But we also have that $\left(x_{n_{k}}(z)\right)_{k}$ converges strongly in $L^{2}$ to $u$. Hence $u(z)=y(z)$ and $u(z) \in H^{2}$.

Moreover, for all $z \in[0,1]$

$$
\|u(z)\|_{H^{2}} \leq \liminf _{k \rightarrow \infty}\left\|x_{n_{k}}(z)\right\|_{H^{2}} \leq C,
$$

by Lemma 3.3 where $C$ does not depend on $z$, so

$$
\sup _{z \in[0,1]}\|u(z, \cdot)\|_{H^{2}}<\infty .
$$

that concludes the proof of (3.7). The proof of (3.8) is a direct consequence of (3.7) and the boundedness of $g^{\prime}$ and $g^{\prime \prime}$.

The second important result of this section is the continuity of the solution of (3.2) with respect to the noise $w$ (we call this map the Itô map). Here we use the notation $X_{w}\left(z_{0}, z\right)$ for the linear flow and $u_{w}$ for the solution of (3.2) driven by $w$. 
Theorem 3.7. Let $u_{0} \in H^{2}$. The Itô map $\mathcal{I}$ associated to (3.2):

$$
\begin{gathered}
\mathcal{I}: \mathcal{C}([0,1], \mathbb{R}) \rightarrow \mathcal{C}\left([0,1], L^{2}\right) \\
\quad w \longmapsto u_{w},
\end{gathered}
$$

is continuous. In fact, it is a Lipschitz map.

Proof. Let $w_{1}$ and $w_{2}$ be two continuous noises. We have

$$
\begin{aligned}
u_{w_{1}}(z, t)-u_{w_{2}}(z, t)= & X_{w_{1}}(0, z) u_{0}(t)+i \int_{0}^{z} X_{w_{1}}\left(z^{\prime}, z\right) g\left(u_{w_{1}}\left(z^{\prime}, t\right)\right) d z^{\prime} \\
& -X_{w_{2}}(0, z) u_{0}(t)-i \int_{0}^{z} X_{w_{2}}\left(z^{\prime}, z\right) g\left(u_{w_{2}}\left(z^{\prime}, t\right)\right) d z^{\prime} \\
= & X_{w_{1}}(0, z) u_{0}(t)-X_{w_{2}}(0, z) u_{0}(t) \\
& +i \int_{0}^{z}\left(X_{w_{1}}\left(z^{\prime}, z\right)-X_{w_{2}}\left(z^{\prime}, z\right)\right) g\left(u_{w_{1}}\left(z^{\prime}, t\right)\right) d z^{\prime} \\
& +i \int_{0}^{z} X_{w_{2}}\left(z^{\prime}, z\right)\left(g\left(u_{w_{1}}\left(z^{\prime}, t\right)\right)-g\left(u_{w_{2}}\left(z^{\prime}, t\right)\right)\right) d z^{\prime},
\end{aligned}
$$

so,

$$
\begin{aligned}
\left\|u_{w_{1}}(z)-u_{w_{2}}(z)\right\|_{L^{2}} \leq & \left\|X_{w_{1}}(0, z) u_{0}-X_{w_{2}}(0, z) u_{0}\right\|_{L^{2}} \\
& +\int_{0}^{z}\left\|\left(X_{w_{1}}\left(z^{\prime}, z\right)-X_{w_{2}}\left(z^{\prime}, z\right)\right) g\left(u_{w_{1}}\left(z^{\prime}\right)\right)\right\|_{L^{2}} d z^{\prime} \\
& +\int_{0}^{z}\left\|g\left(u_{w_{1}}\left(z^{\prime}\right)\right)-g\left(u_{w_{2}}\left(z^{\prime}\right)\right)\right\|_{L^{2}} d z^{\prime} \\
\leq & \left\|w_{1}-w_{2}\right\|_{\infty}\left\|u_{0}\right\|_{H^{2}} \\
& +2\left\|w_{1}-w_{2}\right\|_{\infty} \int_{0}^{z}\left\|g\left(u_{w_{1}}\left(z^{\prime}\right)\right)\right\|_{H^{2}} d z^{\prime} \\
& +\left\|g^{\prime}\right\|_{\infty} \int_{0}^{z}\left\|u_{w_{1}}\left(z^{\prime}\right)-u_{w_{2}}\left(z^{\prime}\right)\right\|_{L^{2}} d z^{\prime} \\
\leq & \left\|w_{1}-w_{2}\right\|_{\infty}\left\|u_{0}\right\|_{H^{2}} \\
& +2 C\left\|w_{1}-w_{2}\right\|\left\|_{\infty}\right\| u_{0} \|_{H^{2}} \\
& +\left\|g^{\prime}\right\|_{\infty} \int_{0}^{z}\left\|u_{w_{1}}\left(z^{\prime}\right)-u_{w_{2}}\left(z^{\prime}\right)\right\|_{L^{2}} d z^{\prime},
\end{aligned}
$$

hence, by Gronwall's lemma,

$$
\sup _{z \in[0,1]}\left\|u_{w_{1}}(z)-u_{w_{2}}(z)\right\|_{L^{2}} \leq 3 C \exp \left(C_{g}\right)\left\|w_{1}-w_{2}\right\|_{\infty},
$$

which concludes the proof.

3.2. Pseudo-spectral scheme. We introduce a pseudo-spectral scheme to solve $(3.2)$ numerically for $(z, t) \in[0,1] \times \mathbb{R}$. We give the definition of the flow $Y(z)$ associated to the equation

$$
i \frac{\partial v}{\partial z}+f\left(|v|^{2}\right) v=0
$$

We consider an initial condition $u_{0} \in L^{2}$. There exists a unique solution in $\mathcal{C}\left([0,1], L^{2}\right)$ to Equation (3.9). This solution can be written as

$$
Y(z) u_{0}(t)=u_{0}(t) \exp \left(i z f\left(\left|u_{0}(t)\right|^{2}\right)\right) .
$$


We can deduce $\left\|Y(z) u_{0}\right\|_{L^{2}}=\left\|u_{0}\right\|_{L^{2}}$.

We let $X_{n}^{h}:=X((n-1) h, n h)$. We fix an integer $N$ and we let $h=1 / N$. We define the function $t \mapsto u_{n}^{h}(t)$ for all $0 \leq n \leq N$ as an approximation of $t \mapsto u(n h, t)$. We begin by $u_{0}^{h}=u_{0}$. Recursively we let for every $n \in\{0, \cdots, N-1\}$ :

$$
u_{n}^{h} \rightarrow u_{n+1}^{h}:\left\{\begin{array}{l}
u_{n+1 / 2}^{h}:=X(n h,(n+1) h) u_{n}^{h}, \\
u_{n+1}^{h}:=Y(h) u_{n+1 / 2}^{h} .
\end{array}\right.
$$

The main result of this section is the following:

TheOREM 3.8. We assume that $u_{0} \in H^{2}$ and there exist two constants $c, \alpha>0$ such that for every $z_{0}, z \in[0,1]$

$$
\sup _{z^{\prime} \in\left[z_{0}, z\right]}\left|w\left(z^{\prime}\right)-w\left(z_{0}\right)\right| \leq c\left|z-z_{0}\right|^{\alpha} .
$$

Then there exists a constant $C$ which depends only on $g, c$ and $\left\|u_{0}\right\|_{H^{2}}$, such that for every $h \in(0,1]$, and $n$ satisfying $n h \leq 1$ :

$$
\left\|u_{n}^{h}-u(n h, \cdot)\right\|_{L^{2}} \leq C h^{\alpha} .
$$

The proof of Theorem 3.8 will be delayed until several technical lemmas can be stated.

We recall and introduce some notation. We respectively denote by $S\left(z_{0}, z\right)$, $X\left(z_{0}, z\right)$ and $Y\left(z-z_{0}\right)$ the flows associated to Equations (3.2), (3.3) and (3.9) with initial conditions in $z_{0}$ and $Z\left(z_{0}, z\right):=Y\left(z-z_{0}\right) X\left(z_{0}, z\right)$. We let $S_{n}^{h}:=S((n-1) h, n h)$, $Z_{n}^{h}:=Z((n-1) h, n h), \widetilde{S}_{n}^{h}:=S_{n}^{h} \cdots S_{1}^{h}$ and $\widetilde{Z}_{n}^{h}:=Z_{n}^{h} \cdots Z_{1}^{h}$. We establish a first lemma.

Lemma 3.9. There exists a constant $c_{0}$ which depends only on $g$ such that for all $u_{1}$ and $u_{2}$ in $H^{2}$ :

$$
\left\|Z\left(z_{0}, z\right) u_{1}-Z\left(z_{0}, z\right) u_{2}\right\|_{L^{2}} \leq\left(1+c_{0}\left(z-z_{0}\right)\right)\left\|u_{1}-u_{2}\right\|_{L^{2}} .
$$

Proof. $X\left(z_{0}, z\right)$ is unitary and linear in $L^{2}$, so it is sufficient to show that

$$
\left\|Y(z) u_{1}-Y(z) u_{2}\right\|_{L^{2}} \leq\left(1+c_{0} z\right)\left\|u_{1}-u_{2}\right\|_{L^{2}} .
$$

We have

$$
Y(z) u_{1}=u_{1}+i \int_{0}^{z} g\left(Y\left(z^{\prime}\right) u_{1}\right) d z^{\prime} .
$$

Using the fact that $g$ is a Lipschitz function:

$$
\left\|Y(z) u_{1}-Y(z) u_{2}\right\|_{L^{2}} \leq\left\|u_{1}-u_{2}\right\|_{L^{2}}+C \int_{0}^{z}\left\|Y\left(z^{\prime}\right) u_{1}-Y\left(z^{\prime}\right) u_{2}\right\|_{L^{2}} d z^{\prime},
$$

where $C$ only depends on $g$. By Gronwall's lemma:

$$
\left\|Y(z) u_{1}-Y(z) u_{2}\right\|_{L^{2}} \leq \exp (C z)\left\|u_{1}-u_{2}\right\|_{L^{2}} .
$$


There exists $c_{0}>0$ such that $\exp (C z) \leq\left(1+c_{0} z\right)$ for every $z \in[0,1]$, so

$$
\left\|Y(z) u_{1}-Y(z) u_{2}\right\|_{L^{2}} \leq\left(1+c_{0} z\right)|| u_{1}-u_{2} \|_{L^{2}},
$$

which completes the proof.

Lemma 3.10. Let $u_{1} \in H^{2}$. We have

$$
\left\|X\left(z_{0}, z\right) u_{1}-u_{1}\right\|_{L^{2}} \leq\left|w(z)-w\left(z_{0}\right)\right|\left\|u_{1}\right\|_{H^{2}} .
$$

Proof. Let $\widehat{u_{1}}$ be the Fourier transform of $u_{1}$. We use the basic estimate $\mid \exp (i x)-$ $\left.1\right|^{2} \leq x^{2}$ to obtain:

$$
\begin{aligned}
\left\|X\left(z_{0}, z\right) u_{1}-u_{1}\right\|_{L^{2}}^{2} & =\int_{-\infty}^{+\infty}\left|\widehat{u_{1}}(\theta) \exp \left(-i \theta^{2}\left(w(z)-w\left(z_{0}\right)\right)\right)-\widehat{u_{1}}(\theta)\right|^{2} d \theta \\
& \leq\left(w(z)-w\left(z_{0}\right)\right)^{2}\left\|u_{1}\right\|_{H^{2}}^{2} .
\end{aligned}
$$

This concludes the proof.

Lemma 3.11. Let $u_{1} \in H^{2}$. There exists a constant $C_{0}$ which depends only on $\left\|u_{1}\right\|_{H^{2}}$ and $g$ such that for every $z \in\left[z_{0}, 1\right]$ :

$$
\left\|S\left(z_{0}, z\right) u_{1}-Z\left(z_{0}, z\right) u_{1}\right\|_{L^{2}} \leq C_{0} \sup _{z^{\prime} \in\left[z_{0}, z\right]}\left|w(z)-w\left(z_{0}\right)\right|\left(z-z_{0}\right) .
$$

Proof. We have

$$
S\left(z_{0}, z\right) u_{1}-Z\left(z_{0}, z\right) u_{1}=R_{1}\left(z_{0}, z\right)+R_{2}\left(z_{0}, z\right),
$$

where

$$
\begin{aligned}
& R_{1}\left(z_{0}, z\right)=i \int_{z_{0}}^{z} X\left(z^{\prime}, z\right)\left\{g\left(S\left(z_{0}, z^{\prime}\right) u_{1}\right)-g\left(Z\left(z_{0}, z^{\prime}\right) u_{1}\right)\right\} d z^{\prime} \\
& R_{2}\left(z_{0}, z\right)=i \int_{z_{0}}^{z}\left\{X\left(z^{\prime}, z\right) g\left(Z\left(z_{0}, z^{\prime}\right) u_{1}\right)-g\left(Y\left(z^{\prime}-z_{0}\right) X\left(z_{0}, z\right) u_{1}\right)\right\} d z^{\prime} .
\end{aligned}
$$

We deal separately with $R_{1}\left(z_{0}, z\right)$ and $R_{2}\left(z_{0}, z\right) . X\left(z^{\prime}, z\right)$ is unitary and $g$ is a Lipschitz function, then

$$
\left\|R_{1}\left(z_{0}, z\right)\right\|_{L^{2}} \leq\left\|g^{\prime}\right\|_{\infty} \int_{z_{0}}^{z}\left\|S\left(z_{0}, z^{\prime}\right) u_{1}-Z\left(z_{0}, z^{\prime}\right) u_{1}\right\|_{L^{2}} d z^{\prime} .
$$

By the fact that $g$ is a Lipschitz function and using Lemmas 3.10 and 3.9:

$$
\begin{aligned}
\left\|R_{2}\left(z_{0}, z\right)\right\|_{L^{2}} \leq & \int_{z_{0}}^{z}\left\|X\left(z^{\prime}, z\right) g\left(Z\left(z_{0}, z^{\prime}\right) u_{1}\right)-g\left(Z\left(z_{0}, z^{\prime}\right) u_{1}\right)\right\|_{L^{2}} d z^{\prime} \\
& +\int_{z_{0}}^{z}\left\|g\left(Z\left(z_{0}, z^{\prime}\right) u_{1}\right)-g\left(Y\left(z^{\prime}-z_{0}\right) X\left(z_{0}, z\right) u_{1}\right)\right\|_{L^{2}} d z^{\prime} \\
\leq & C \sup _{z^{\prime} \in\left[z_{0}, z\right]}\left|w\left(z^{\prime}\right)-w\left(z_{0}\right)\right| \int_{z_{0}}^{z}\left\|Z\left(z_{0}, z^{\prime}\right) u_{1}\right\|_{H^{2}} d z^{\prime} \\
& +\left\|g^{\prime}\right\|_{\infty} \int_{z_{0}}^{z}\left(1+c_{0}\left(z^{\prime}-z_{0}\right)\right)\left\|X\left(z_{0}, z^{\prime}\right) u_{1}-X\left(z_{0}, z\right) u_{1}\right\|_{L^{2}} d z^{\prime} \\
\leq & C\left(z-z_{0}\right) \sup _{z^{\prime} \in\left[z_{0}, z\right]}\left|w\left(z^{\prime}\right)-w\left(z_{0}\right)\right|,
\end{aligned}
$$


where the last constant $C$ depends on $g$ and the $H^{2}$-norm of $u_{1}$. So, following (3.11) there exists a constant $C$ depending on $g$ and the $H^{2}$-norm of $u_{1}$ such that for every $z_{0}, z_{1}$ and $z$ satisfying $z_{0} \leq z_{1} \leq z$ :

$$
\begin{aligned}
\left\|S\left(z_{0}, z_{1}\right) u_{1}-Z\left(z_{0}, z_{1}\right) u_{1}\right\|_{L^{2}} \leq & C\left(z_{1}-z_{0}\right) \sup _{z^{\prime} \in\left[z_{0}, z\right]}\left|w\left(z^{\prime}\right)-w\left(z_{0}\right)\right| \\
& +C \int_{z_{0}}^{z_{1}}\left\|S\left(z_{0}, z^{\prime}\right) u_{1}-Z\left(z_{0}, z^{\prime}\right) u_{1}\right\|_{L^{2}} d z^{\prime} .
\end{aligned}
$$

The proof is completed by an application of Gronwall's lemma.

Lemma 3.12. Let $u_{1}$ in $H^{2}$. There exists a constant $C$ which depends only on $g$, $\left\|u_{1}\right\|_{H^{2}}$ such that for every $z \in\left[z_{0}, 1\right]$ :

$$
\left\|S\left(z_{0}, z\right) u_{1}-Z\left(z_{0}, z\right) u_{1}\right\|_{L^{2}} \leq C\left(z-z_{0}\right)^{1+\alpha} .
$$

Proof. This is a direct consequence of Lemma 3.11 and (3.10).

Now we prove Theorem 3.8.

Proof. (Theorem 3.8) We use the telescopic sum

$$
\begin{aligned}
u_{n}^{h}-u(n h, \cdot) & =Z_{n}^{h} \cdots Z_{1}^{h} u_{0}-S_{n}^{h} \cdots S_{1}^{h} u_{0} \\
& =\sum_{j=1}^{n}\left\{Z_{n}^{h} \cdots Z_{j}^{h} S_{j-1}^{h} \cdots S_{1}^{h} u_{0}-Z_{n}^{h} \cdots Z_{j+1}^{h} S_{j}^{h} \cdots S_{1}^{h} u_{0}\right\} .
\end{aligned}
$$

Taking $L^{2}$-norm and applying Lemma 3.9 we obtain existence of a constant $c_{0}$ such that

$$
\left\|u_{n}^{h}-u(n h, \cdot)\right\|_{L^{2}} \leq \sum_{j=1}^{n}\left(1+c_{0} h\right)^{n-j}\left\|\left(Z_{j}^{h}-S_{j}^{h}\right) S_{j-1}^{h} \cdots S_{1}^{h} u_{0}\right\|_{L^{2}}
$$

Using Lemma 3.12 and the uniform boundedness of $S\left(z_{0}, z\right)$ in $H^{2}$, there exists $C$ such that

$$
\left\|u_{n}^{h}-u(n h, \cdot)\right\|_{L^{2}} \leq C \sum_{j=1}^{n}\left(1+c_{0} h\right)^{(n-j)} h^{1+\alpha} \leq C \exp \left(c_{0}\right) h^{\alpha} .
$$

The proof is completed.

4. Error estimate of the pseudo-spectral scheme for the electric field

This section deals with the numerical study of the electric field $u^{\varepsilon}(z, t)=u\left(z / \varepsilon^{2}, t\right)$ which is solution of

$$
i \frac{\partial u^{\varepsilon}}{\partial z}+\frac{1}{\varepsilon} m\left(\frac{z}{\varepsilon^{2}}\right) \frac{\partial^{2} u^{\varepsilon}}{\partial t^{2}}+f\left(\left|u^{\varepsilon}\right|^{2}\right) u^{\varepsilon}=0 .
$$

4.1. The split-step scheme. As for (3.2), there exists a unique solution of $u^{\varepsilon}$ in $\mathcal{C}\left([0,1], L^{2}\right)$ if we take $u_{0} \in L^{2}$. We denote by $S^{\varepsilon}\left(z_{0}, z\right)$ the flow associated to the problem (4.1) with initial condition in $z=z_{0}$. 
We introduce the split-step scheme to solve (4.1) numerically for $(z, t) \in[0,1] \times \mathbb{R}$. We consider the problems:

$$
\begin{aligned}
i \frac{\partial v^{\varepsilon}}{\partial z}+\frac{1}{\varepsilon} m\left(\frac{z}{\varepsilon^{2}}\right) \frac{\partial^{2} v^{\varepsilon}}{\partial t^{2}} & =0 \\
i \frac{\partial v}{\partial z}+f\left(|v|^{2}\right) v & =0 .
\end{aligned}
$$

We denote respectively $X^{\varepsilon}\left(z_{0}, z\right)$ and $Y\left(z-z_{0}\right)$ the flows associated to Equations (4.2) and (4.3) with initial condition at $z_{0}$. Moreover we let $Z^{\varepsilon}\left(z_{0}, z\right):=Y\left(z-z_{0}\right) X^{\varepsilon}\left(z_{0}, z\right)$.

We let $X_{n}^{\varepsilon, h}:=X^{\varepsilon}((n-1) h, n h)$. We fix an integer $N$ and we let $h=1 / N$. We define $u_{n}^{\varepsilon, h}$ for every $0 \leq n \leq N$ as an approximation of $u^{\varepsilon}(n h, \cdot)$. We begin by $u_{0}^{\varepsilon, h}=u_{0}$. Then we define recursively for every $n \in\{0, \cdots, N-1\}$ :

$$
u_{n}^{\varepsilon, h} \rightarrow u_{n+1}^{\varepsilon, h}:\left\{\begin{array}{l}
u_{n+1 / 2}^{\varepsilon, h}:=X^{\varepsilon}(n h,(n+1) h) u_{n}^{\varepsilon, h}, \\
u_{n+1}^{\varepsilon, h}:=Y(h) u_{n+1 / 2}^{\varepsilon, h}
\end{array} .\right.
$$

$u^{\varepsilon, h}$ is defined by: for every $z \in[0,1]$,

$$
u^{\varepsilon, h}(z)=\sum_{j=0}^{N-1} \mathbb{I}_{[j h,(j+1) h)}(z)\left(u_{j}^{\varepsilon, h}+(z / h-[z / h])\left(u_{j+1}^{\varepsilon, h}-u_{j}^{\varepsilon, h}\right)\right),
$$

where $\mathbb{I}_{A}(z)=1$ if $z \in A$ and 0 otherwise. Finally we let $S_{n}^{\varepsilon, h}:=S^{\varepsilon}((n-1) h, n h)$, $Z_{n}^{\varepsilon, h}:=Z^{\varepsilon}((n-1) h, n h)$.

4.2. Error estimate. The main result of this section is the following.

THEOREM 4.1. We assume that $u_{0} \in H^{2}$. There exists an constant $C$ depending only on $g$ and $\left\|u_{0}\right\|_{H^{2}}$, such that for every $\left.\left.\varepsilon<1, h \in\right] 0,1\right]$, and $n$ satisfying $n h \leq 1$ :

$$
\mathbb{E}\left[\| u_{n}^{\varepsilon, h}-\left.u^{\varepsilon}(n h, \cdot)\right|_{L^{2}} ^{2}\right] \leq C\left(h+\varepsilon^{2}\right) .
$$

Moreover, for every $z \in[0,1]$ :

$$
\mathbb{E}\left[\left\|u^{\varepsilon, h}(z)-u^{\varepsilon}(z, \cdot)\right\|_{L^{2}}^{2}\right] \leq C\left(h+\varepsilon^{2}\right) .
$$

Note that in classical simulation situations, the numerical grid step is smaller than the smallest scale of the problem. Here, we can choose a numerical grid step which is larger than the smallest scale, the natural scale of variations of the random coefficient, because the solution varies relatively slowly.

The proof of this result follows the same lines as the proof of Theorem 3.8 and use strongly the results of Section 3 and the following remark.

REMARK 4.2. Lemmas 3.9, 3.10 and 3.11 establish properties of the operators $X\left(z_{0}, z\right), Z\left(z_{0}, z\right)$ and $S\left(z_{0}, z\right)$ defined in Section 3 with an arbitrary continuous noise $w$. If we consider the noise $w(z)=w^{\varepsilon}(z)=\int_{0}^{z}(1 / \varepsilon) m\left(z^{\prime} / \varepsilon^{2}\right) d z^{\prime}$, these operators are respectively $X^{\varepsilon}\left(z_{0}, z\right), Z^{\varepsilon}\left(z_{0}, z\right)$ and $S^{\varepsilon}\left(z_{0}, z\right)$ defined in this section. Hence, Lemmas 3.9, 3.10 and 3.11 can be used here. Moreover, the constants which appear in Lemmas 3.9 and 3.11 are independant on $\varepsilon$ because they are independant on the noise $w$.

We also need the two following lemmas. 
Lemma 4.3. For every $l \in \mathbb{N}^{*}$, there exists a constant $C_{l}$ such that

$$
\mathbb{E}\left[\left(\sup _{z^{\prime} \in\left[z_{0}, z\right]}\left|\int_{z_{0}}^{z^{\prime}} \frac{1}{\varepsilon} m\left(\frac{z^{\prime \prime}}{\varepsilon^{2}}\right) d z^{\prime \prime}\right|\right)^{2 l}\right] \leq C_{l}\left(\varepsilon^{2 l}+\left(z-z_{0}\right)^{l}\right) .
$$

Proof. We denote by $\mathcal{L}$ the infinitesimal generator of $m$. Following the Fredholm alternative, there exists a function $f_{1}(m)$ such that $\mathcal{L} f_{1}(m)=m$. Moreover, the process $M_{z_{0}}$ defined by

$$
M_{z_{0}}(z)=f_{1}(m(z))-f_{1}\left(m\left(z_{0}\right)\right)-\int_{z_{0}}^{z} \mathcal{L} f_{1}\left(m\left(z^{\prime}\right)\right) d z^{\prime}
$$

is a martingale with bracket

$$
\left\langle M_{z_{0}}\right\rangle(z)=\int_{z_{0}}^{z}\left(\mathcal{L}\left(f_{1}^{2}\right)\left(m\left(z^{\prime}\right)\right)-2 f_{1} \mathcal{L} f_{1}\left(m\left(z^{\prime}\right)\right)\right) d z^{\prime} .
$$

So we have

$$
\int_{z_{0}}^{z^{\prime}} \frac{1}{\varepsilon} m\left(\frac{z^{\prime \prime}}{\varepsilon^{2}}\right) d z^{\prime \prime}=\varepsilon\left(f_{1}\left(m\left(z^{\prime} / \varepsilon^{2}\right)\right)-f_{1}\left(m\left(z_{0} / \varepsilon^{2}\right)\right)\right)-\varepsilon M_{z_{0} / \varepsilon^{2}}\left(z^{\prime} / \varepsilon^{2}\right) .
$$

By Burkholder's inequality and the boundedness of $m$, there exists $C_{l}$ such that

$$
\begin{aligned}
\mathbb{E}\left[\sup _{z^{\prime} \in\left[z_{0}, z\right]}\left|\varepsilon M_{z_{0} / \varepsilon^{2}}\left(z^{\prime} / \varepsilon^{2}\right)\right|^{2 l}\right] & \leq \varepsilon^{2 l} C \mathbb{E}\left[\left\langle M_{z_{0} / \varepsilon^{2}}\right\rangle\left(z / \varepsilon^{2}\right)^{l}\right] \\
& \leq \varepsilon^{2 l} C_{l} \mathbb{E}\left[\left(\int_{z_{0} / \varepsilon^{2}}^{z / \varepsilon^{2}}\left(\mathcal{L}\left(f_{1}^{2}\right)\left(m\left(z^{\prime}\right)\right)-2 f_{1} \mathcal{L} f_{1}\left(m\left(z^{\prime}\right)\right)\right) d z^{\prime}\right)\right] \\
& \leq \varepsilon^{2 l} C_{l}\left(\frac{z}{\varepsilon^{2}}-\frac{z_{0}}{\varepsilon^{2}}\right)^{l}=C_{l}\left(z-z_{0}\right)^{l} .
\end{aligned}
$$

Moreover, again by the boundedness of $m$, and the Doeblin's condition, there exists $C_{l}^{\prime}$ such that

$$
\mathbb{E}\left[\sup _{z^{\prime} \in\left[z_{0}, z\right]}\left|\varepsilon\left(f_{1}\left(m\left(z^{\prime} / \varepsilon^{2}\right)\right)-f_{1}\left(m\left(z_{0} / \varepsilon^{2}\right)\right)\right)\right|^{2 l}\right] \leq \varepsilon^{2 l} C_{l}^{\prime} .
$$

Using (4.4) and (4.5) we complete the proof.

Lemma 4.4. Let $u_{0} \in H^{2}$. There exists $C$ depending only on $g$ and $\left\|u_{0}\right\|_{H^{2}}$ such that for every $h \in(0,1)$ and $n \in \mathbb{N}$ such that $n h \leq 1$,

$$
\left\|Z_{n}^{\varepsilon, h} Z_{n-1}^{\varepsilon, h} \cdots Z_{1}^{\varepsilon, h} u_{0}\right\|_{H^{2}} \leq C
$$

Proof. Let $u_{0} \in H^{2}, h \in(0,1)$ and $n \in \mathbb{N} . X\left(z_{0}, z\right)$ and $Y\left(z-z_{0}\right)$ are unitary in $L^{2}$, so, we have

$$
\left\|Z_{n}^{\varepsilon, h} Z_{n-1}^{\varepsilon, h} \cdots Z_{1}^{\varepsilon, h} u_{0}\right\|_{L^{2}}=\left\|u_{0}\right\|_{L^{2}},
$$


that is to say $Z_{n}^{\varepsilon, h} Z_{n-1}^{\varepsilon, h} \cdots Z_{1}^{\varepsilon, h}$ is unitary in $L^{2}$.

Let $u_{1} \in H^{1}$. We have

$$
\left\|\partial_{t} Y(z) u_{1}\right\|_{L^{2}} \leq\left\|\partial_{t} u_{1}\right\|_{L^{2}}+\left\|g^{\prime}\right\|_{\infty} \int_{0}^{z}\left\|\partial_{t} Y\left(z^{\prime}\right) u_{1}\right\|_{L^{2}} d z^{\prime}
$$

By Gronwall's lemma, there exists $C$ depending on $g$ such that for every $h$ :

$$
\left\|Y(h) u_{1}\right\|_{H^{1}} \leq\left\|u_{1}\right\|_{H^{1}} \exp (C h) .
$$

So, $X\left(z_{0}, z\right)$ being unitary in $H^{1}$, we have with the same constant $C$ as previously

$$
\left\|Z_{n}^{\varepsilon, h} Z_{n-1}^{\varepsilon, h} \cdots Z_{1}^{\varepsilon, h} u_{0}\right\|_{H^{1}} \leq\left\|u_{0}\right\|_{H^{1}} \exp (C n h) \leq\left\|u_{0}\right\|_{H^{1}} \exp (C) .
$$

Let $u_{1} \in H^{2}$. We have

$$
\begin{aligned}
\left\|\partial_{t}^{2} Y(z) u_{1}\right\|_{L^{2}} \leq & \left\|\partial_{t}^{2} u_{1}\right\|_{L^{2}} \\
& +\left\|g^{\prime}\right\|_{\infty} \int_{0}^{z}\left\|\partial_{t}^{2} Y\left(z^{\prime}\right) u_{1}\right\|_{L^{2}} d z^{\prime} \\
& +\left\|g^{\prime \prime}\right\|_{\infty} \int_{0}^{z}\left\|\partial_{t} Y\left(z^{\prime}\right) u_{1}\right\|_{L^{4}}^{2} d z^{\prime}
\end{aligned}
$$

Hence, by Gagliardo-Nirenberg's inequality $\|\cdot\|_{L^{4}}^{2} \leq C_{g n}\|\cdot\|\left\|_{L^{2}}\right\| \cdot \|_{H^{1}}$ and the previous step of the proof, there exists $C$ depending on $g$ and $\left\|u_{1}\right\|_{H^{1}}$ such that:

$$
\left\|Y(z) u_{1}\right\|_{H^{2}} \leq\left\|u_{1}\right\|_{H^{2}}+C \int_{0}^{z}\left\|Y\left(z^{\prime}\right) u_{1}\right\|_{H^{2}} d z^{\prime}
$$

So by Gronwall's lemma and the identity $\left\|\partial_{t}^{2} X\left(z_{0}, z\right) u_{1}\right\|_{L^{2}}=\left\|\partial_{t}^{2} u_{1}\right\|_{L^{2}}$, there exists $C^{\prime}$ depending on $g$ and $\left\|u_{1}\right\|_{H^{1}}$ such that for every $j \leq n$ :

$$
\left\|Z_{j}^{\varepsilon, h} u_{1}\right\|_{H^{2}} \leq\left\|u_{1}\right\|_{H^{2}} \exp \left(C^{\prime} h\right) .
$$

Moreover, $\left\|Z_{n}^{\varepsilon, h} Z_{n-1}^{\varepsilon, h} \cdots Z_{1}^{\varepsilon, h} u_{0}\right\|_{H^{1}} \leq\left\|u_{0}\right\|_{H^{1}} \exp (C)$, so there exists $C^{\prime \prime}$ depending on $g$ and $\left\|u_{0}\right\|_{H^{1}}$ such that

$$
\left\|Z_{n}^{\varepsilon, h} Z_{n-1}^{\varepsilon, h} \cdots Z_{1}^{\varepsilon, h} u_{0}\right\|_{H^{2}} \leq\left\|u_{0}\right\|_{H^{2}} \exp \left(C^{\prime \prime} n h\right) \leq\left\|u_{0}\right\|_{H^{2}} \exp \left(C^{\prime \prime}\right) .
$$

The proof is completed.

Now we prove Theorem 4.1.

Proof. (Theorem 4.1) We use the telescopic sum

$$
\begin{aligned}
u_{n}^{\varepsilon, h}-u^{\varepsilon}(n h, \cdot) & =Z_{n}^{\varepsilon, h} \cdots Z_{1}^{\varepsilon, h} u_{0}-S_{n}^{\varepsilon, h} \cdots S_{1}^{\varepsilon, h} u_{0} \\
& =\sum_{j=1}^{n}\left\{Z_{n}^{\varepsilon, h} \cdots Z_{j}^{\varepsilon, h} S_{j-1}^{\varepsilon, h} \cdots S_{1}^{\varepsilon, h} u_{0}-Z_{n}^{\varepsilon, h} \cdots Z_{j+1}^{\varepsilon, h} S_{j}^{\varepsilon, h} \cdots S_{1}^{\varepsilon, h} u_{0}\right\} .
\end{aligned}
$$

We take the $L^{2}$-norm. Then, by Lemma 3.9, there exists a constant $c_{0}$ such that

$$
\left\|u_{n}^{\varepsilon, h}-u^{\varepsilon}(n h, \cdot)\right\|_{L^{2}} \leq \sum_{j=1}^{n}\left(1+c_{0} h\right)^{n-j}\left\|\left(Z_{j}^{\varepsilon, h}-S_{j}^{\varepsilon, h}\right) S_{j-1}^{\varepsilon, h} \cdots S_{1}^{\varepsilon, h} u_{0}\right\|_{L^{2}} .
$$


We take the expectation, we use Lemmas 3.11 and 4.3 and the fact that $S^{\varepsilon}\left(z_{0}, z\right)$ is uniformly bounded in $H^{2}$. So there exists $C$ such that

$$
\mathbb{E}\left[\| u_{n}^{\varepsilon, h}-\left.u^{\varepsilon}(n h, \cdot)\right|_{L^{2}} ^{2}\right] \leq C n \sum_{j=1}^{n}\left(1+c_{0} h\right)^{2(n-j)}\left(h^{3}+\varepsilon^{2} h^{2}\right) \leq C \exp \left(2 c_{0}\right)\left(h+\varepsilon^{2}\right) .
$$

We get the first part of Theorem 4.1. For the second part, it is sufficient to show that for every $z \in[n h,(n+1) h)$

$$
\begin{array}{ll} 
& \mathbb{E}\left[\left\|u^{\varepsilon}(z, \cdot)-u^{\varepsilon}(n h, \cdot)\right\|_{L^{2}}^{2}\right] \leq C\left(h+\varepsilon^{2}\right) \\
\text { and } \quad & \mathbb{E}\left[\left\|u^{\varepsilon, h}(z, \cdot)-u_{n}^{\varepsilon, h}(\cdot)\right\|_{L^{2}}^{2}\right] \leq C\left(h+\varepsilon^{2}\right) .
\end{array}
$$

For (4.6), we have

$$
\begin{aligned}
u^{\varepsilon}(z, \cdot)-u^{\varepsilon}(n h, \cdot)= & X^{\varepsilon}(n h, z) u^{\varepsilon}(n h, \cdot)-u^{\varepsilon}(n h, \cdot) \\
& +i \int_{n h}^{z} X^{\varepsilon}\left(z^{\prime}, z\right) g\left(u^{\varepsilon}\left(z^{\prime}, \cdot\right)\right) d z^{\prime} .
\end{aligned}
$$

Now we take the $L^{2}-$ norm:

$$
\begin{aligned}
\left\|u^{\varepsilon}(z, \cdot)-u^{\varepsilon}(n h, \cdot)\right\|_{L^{2}} \leq & \left\|X^{\varepsilon}(n h, z) u^{\varepsilon}(n h, \cdot)-u^{\varepsilon}(n h, \cdot)\right\|_{L^{2}} \\
& +C(z-n h)\left\|u_{0}(\cdot)\right\|_{L^{2}} .
\end{aligned}
$$

In applying Lemmas 3.10 and 4.3 and $|z-n h| \leq h$ we obtain (4.6). For (4.7), we use Lemmas 3.10, 4.3 and 4.4.

By a slight modification of the proof, we can obtain an extension of the previous result. This extension will be used to establish a uniform error estimate.

Lemma 4.5. Let $u_{0} \in H^{2}$. There exists $C$ depending only on $g$ and $\left\|u_{0}\right\|_{H^{2}}$ such that for every $\varepsilon<1, h \in(0,1]$, and $n$ such that $n h \leq 1$, for every $z \in[0,1]$ :

$$
\mathbb{E}\left[\left\|u^{\varepsilon, h}(z)-u^{\varepsilon}(z, \cdot)\right\|_{L^{2}}^{4}\right] \leq C\left(h^{2}+\varepsilon^{4}\right) .
$$

4.3. Uniform error estimate. Here we establish the following result.

Theorem 4.6. Let $u_{0} \in H^{2}$. There exists $C$ depending only on $\left\|u_{0}\right\|_{H^{2}}$ such that for every $\varepsilon<1, h \in(0,1]$ and $\gamma>0$ :

$$
\mathbb{P}\left[\sup _{z \in[0,1]}\left\|u^{\varepsilon, h}(z)-u^{\varepsilon}(z)\right\|_{L^{2}}>\gamma\right] \leq \frac{C}{\gamma^{4}}\left(h+\varepsilon^{4} / h\right) .
$$

Proof. We let $v^{\varepsilon, h}(z, t):=u^{\varepsilon, h}(z, t)-u^{\varepsilon}(z, t)$ for every $z$ and $t$. For every $N>0$ :

$$
\begin{aligned}
\mathbb{P}\left[\sup _{z \in[0,1]}\left\|v^{\varepsilon, h}(z)\right\|_{L^{2}}>\gamma\right] & =\mathbb{P}\left[\bigcup_{j=0}^{N-1}\left\{\sup _{z \in[j / N,(j+1) / N]}\left\|v^{\varepsilon, h}(z)\right\|_{L^{2}}>\gamma\right\}\right] \\
& \leq \sum_{j=0}^{N-1} \mathbb{P}\left[\sup _{z \in[j / N,(j+1) / N]}\left\|v^{\varepsilon, h}(z)\right\|_{L^{2}}>\gamma\right] \\
& \leq \sum_{j=0}^{N-1} \mathbb{P}\left[\sup _{z \in[j / N,(j+1) / N]}\left\|v^{\varepsilon, h}(z)-v^{\varepsilon, h}\left(\frac{j}{N}\right)\right\|_{L^{2}}>\frac{\gamma}{2}\right] \\
& +\sum_{j=0}^{N-1} \mathbb{P}\left[\left\|v^{\varepsilon, h}(j / N)\right\|_{L^{2}}>\frac{\gamma}{2}\right] .
\end{aligned}
$$


By Markov's inequality and Lemma 4.5, with $N=1 / h$, we have:

$$
\sum_{j=0}^{N-1} \mathbb{P}\left[\left\|v^{\varepsilon, h}(j / N)\right\|_{L^{2}}>\frac{\gamma}{2}\right] \leq \frac{C}{\gamma^{4}}\left(h+\varepsilon^{4} / h\right) .
$$

By Lemma 4.7 below, with $N=1 / h$, we get:

$$
\sum_{j=0}^{N-1} \mathbb{P}\left[\sup _{z \in[j / N,(j+1) / N]}\left\|v^{\varepsilon, h}(z)-v^{\varepsilon, h}\left(\frac{j}{N}\right)\right\|_{L^{2}}>\frac{\gamma}{2}\right] \leq \frac{C}{\gamma^{4}}\left(h+\varepsilon^{4} / h\right) .
$$

This concludes the proof.

LemMA 4.7. We let $v^{\varepsilon, h}(z, t):=u^{\varepsilon, h}(z, t)-u^{\varepsilon}(z, t)$ for $z$ and $t$. Let $u_{0} \in H^{2}, z, \varepsilon$, and $h$ in $(0,1]$ and $\gamma>0$ then

$$
\mathbb{P}\left[\sup _{z^{\prime} \in[z, z+h]}\left\|v^{\varepsilon, h}\left(z^{\prime}\right)-v^{\varepsilon, h}(z)\right\|_{L^{2}}>\frac{\gamma}{2}\right] \leq \frac{C}{\gamma^{4}}\left(h^{2}+\varepsilon^{4}\right)
$$

Proof. We have

$$
\sup _{[z, z+h]}\left\|v^{\varepsilon, h}-v^{\varepsilon, h}(z)\right\|_{L^{2}} \leq \sup _{[z, z+h]}\left\|u^{\varepsilon, h}-u^{\varepsilon, h}(z)\right\|_{L^{2}}+\sup _{[z, z+h]}\left\|u^{\varepsilon}-u^{\varepsilon}(z)\right\|_{L^{2}}
$$

We begin by dealing with $\sup _{[z, z+h]}\left\|u^{\varepsilon, h}-u^{\varepsilon, h}(z)\right\|_{L^{2}}$. For $z^{\prime \prime} \in[0,1]$ we let:

$$
\widetilde{u}^{\varepsilon, h}\left(z^{\prime \prime}\right)=\sum_{j=0}^{N-1} \mathbb{I}_{[j h,(j+1) h)}\left(z^{\prime \prime}\right) u_{j}^{\varepsilon, h}
$$

If $z^{\prime} \in\left[h[z / h], h[z / h]+h\left[\right.\right.$, then $\left[z^{\prime} / h\right]=[z / h]$ so $\widetilde{u}^{\varepsilon, h}\left(z^{\prime}\right)-\widetilde{u}^{\varepsilon, h}(z)=0$. If $z^{\prime} \in[h[z / h]+$ $h, h[z / h]+2 h\left[\right.$, then $\left[z^{\prime} / h\right]=[z / h]+1$ so

$$
\begin{aligned}
\widetilde{u}^{\varepsilon, h}\left(z^{\prime}\right)-\widetilde{u}^{\varepsilon, h}(z)= & u_{[z / h]+1}^{\varepsilon, h}-u_{[z / h]}^{\varepsilon, h} \\
= & Y(z) X_{[z / h]}^{\varepsilon, h} u_{[z / h]}^{\varepsilon, h}-u_{[z / h]}^{\varepsilon, h} \\
= & Y(z) X_{[z / h]}^{\varepsilon, h} u_{[z / h]}^{\varepsilon, h}-X_{[z / h]}^{\varepsilon, h} u_{[z / h]}^{\varepsilon, h} \\
& +X_{[z / h]}^{\varepsilon, h} u_{[z / h]}^{\varepsilon, h}-u_{[z / h]}^{\varepsilon, h} .
\end{aligned}
$$

On the one hand we have

$$
\begin{aligned}
\left\|Y(z) X_{[z / h]}^{\varepsilon, h} u_{[z / h]}^{\varepsilon, h}-X_{[z / h]}^{\varepsilon, h} u_{[z / h]}^{\varepsilon, h}\right\|_{L^{2}} & \leq \int_{0}^{h}\left\|g\left(Y\left(z^{\prime \prime}\right) X_{[z / h]}^{\varepsilon, h} u_{[z / h]}^{\varepsilon, h}\right)\right\|_{L^{2}} d z^{\prime \prime} \\
& \leq C h,
\end{aligned}
$$

where $C$ depends on $g$ and $u_{0}$. On the other hand,

$$
\left\|X_{[z / h]}^{\varepsilon, h} u_{[z / h]}^{\varepsilon, h}-u_{[z / h]}^{\varepsilon, h}\right\|_{L^{2}} \leq C\left|\int_{h[z / h]}^{h[z / h]+h} \frac{1}{\varepsilon} m\left(\frac{z^{\prime \prime}}{\varepsilon^{2}}\right) d z^{\prime \prime}\right|,
$$


where $C$ depends on $g$ and $u_{0}$ by Lemma 4.4. Then we have

$$
\left\|\widetilde{u}_{[z / h]+1}^{\varepsilon, h}-\widetilde{u}_{[z / h]}^{\varepsilon, h}\right\|_{L^{2}} \leq C\left(\left|\int_{h[z / h]}^{h[z / h]+h} \frac{1}{\varepsilon} m\left(\frac{z^{\prime \prime}}{\varepsilon^{2}}\right) d z^{\prime \prime}\right|+h\right) .
$$

We take the fourth moment and we apply Lemmas 3.10 and 4.3, and we obtain

$$
\mathbb{E}\left[\sup _{z^{\prime} \in[z, z+h]}\left\|\widetilde{u}^{\varepsilon, h}\left(z^{\prime}\right)-\widetilde{u}^{\varepsilon, h}(z)\right\|_{L^{2}}^{4}\right] \leq C\left(h^{2}+\varepsilon^{4}\right) .
$$

It remains to prove

$$
\mathbb{E}\left[\sup _{z^{\prime} \in[z, z+h]}\left\|\widetilde{u}^{\varepsilon, h}\left(z^{\prime}\right)-u^{\varepsilon, h}\left(z^{\prime}\right)\right\|_{L^{2}}^{4}\right] \leq C\left(h^{2}+\varepsilon^{4}\right) .
$$

For every $z^{\prime}$, we have

$$
u^{\varepsilon, h}\left(z^{\prime}\right)-\widetilde{u}^{\varepsilon, h}\left(z^{\prime}\right)=\left(z^{\prime} / h-\left[z^{\prime} / h\right]\right)\left(u_{\left[z^{\prime} / h\right]+1}^{\varepsilon, h}-u_{\left[z^{\prime} / h\right]}^{\varepsilon, h}\right),
$$

hence

$$
\left\|\widetilde{u}^{\varepsilon, h}\left(z^{\prime}\right)-u^{\varepsilon, h}\left(z^{\prime}\right)\right\|_{L^{2}} \leq C\left(\left|\int_{h\left[z^{\prime} / h\right]}^{h\left[z^{\prime} / h\right]+h} \frac{1}{\varepsilon} m\left(\frac{z^{\prime \prime}}{\varepsilon^{2}}\right) d z^{\prime \prime}\right|+h\right),
$$

so,

$$
\begin{aligned}
\sup _{z^{\prime} \in[z, z+h]}\left\|\widetilde{u}^{\varepsilon, h}\left(z^{\prime}\right)-u^{\varepsilon, h}\left(z^{\prime}\right)\right\|_{L^{2}} \leq & C\left(\left|\int_{h[z / h]}^{h[z / h]+h} \frac{1}{\varepsilon} m\left(\frac{z^{\prime \prime}}{\varepsilon^{2}}\right) d z^{\prime \prime}\right|\right. \\
& \left.+\left|\int_{h[z / h]+h}^{h[z / h]+2 h} \frac{1}{\varepsilon} m\left(\frac{z^{\prime \prime}}{\varepsilon^{2}}\right) d z^{\prime \prime}\right|+h\right) .
\end{aligned}
$$

We get (4.8) in taking the expectation and in applying Lemma 4.3. It remains to deal with $\sup _{z^{\prime} \in[z, z+h]}\left\|u^{\varepsilon}\left(z^{\prime}\right)-u^{\varepsilon}(z)\right\|_{L^{2}}$. Let $z^{\prime} \in[z, z+h]$, we have

$$
u^{\varepsilon}\left(z^{\prime}\right)-u^{\varepsilon}(z)=X^{\varepsilon}\left(z, z^{\prime}\right) u^{\varepsilon}(z)-u^{\varepsilon}(z)+i \int_{z}^{z^{\prime}} X^{\varepsilon}\left(z^{\prime \prime}, z^{\prime}\right) g\left(u^{\varepsilon}\left(z^{\prime \prime}\right)\right) d z^{\prime \prime},
$$

there exists $C$ depending on $g$ and $u_{0}$ such that

$$
\begin{aligned}
\left\|u^{\varepsilon}\left(z^{\prime}\right)-u^{\varepsilon}(z)\right\|_{L^{2}} & \leq\left\|X^{\varepsilon}\left(z, z^{\prime}\right) u^{\varepsilon}(z)-u^{\varepsilon}(z)\right\|_{L^{2}}+\int_{z}^{z^{\prime}}\left\|g\left(u^{\varepsilon}\left(z^{\prime \prime}\right)\right)\right\|_{L^{2}} d z^{\prime \prime} \\
& \leq C\left(h+\left|\int_{z}^{z^{\prime}} \frac{1}{\varepsilon} m\left(\frac{z^{\prime \prime}}{\varepsilon^{2}}\right) d z^{\prime \prime}\right|\right) .
\end{aligned}
$$

We take the supremum and the fourth moment and we apply Lemma 4.3. We obtain:

$$
\mathbb{E}\left[\sup _{z^{\prime} \in[z, z+h]}\left\|u^{\varepsilon}\left(z^{\prime}\right)-u^{\varepsilon}(z)\right\|_{L^{2}}^{4}\right] \leq C\left(h^{2}+\varepsilon^{4}\right) .
$$

Then we apply Markov's inequality to complete the proof. 


\section{Diffusion-approximation}

In this section we deal with the effective pulse propagation. We establish diffusionapproximation results for the exact solution and the numerical solution.

5.1. Diffusion-approximation for the exact solution. We consider the equation:

$$
u(z, t)=u_{0}(t)+i \sigma_{0} \int_{0}^{z} \frac{\partial^{2} u}{\partial t^{2}}\left(z^{\prime}, t\right) \circ d W\left(z^{\prime}\right)+i \int_{0}^{z} f\left(\left|u\left(z^{\prime}, t\right)\right|^{2}\right) u\left(z^{\prime}, t\right) d z^{\prime},
$$

where $W$ is a standard Brownian motion and $\sigma_{0}>0$. The mild form of this equation is:

$$
u(z, t)=X(0, z) u_{0}(t)+i \int_{0}^{z} X\left(z^{\prime}, z\right) f\left(\left|u\left(z^{\prime}, t\right)\right|^{2}\right) u\left(z^{\prime}, t\right) d z^{\prime},
$$

where $X\left(z_{0}, z\right)$ is a.s. defined by:

$$
X\left(z_{0}, z\right) v_{0}(t)=\frac{1}{\sqrt{4 i \pi \sigma_{0} W\left(z_{0}, z\right)}} \int_{-\infty}^{\infty} \exp \left(\frac{i\left(t-t^{\prime}\right)^{2}}{4 \sigma_{0} W\left(z_{0}, z\right)}\right) v_{0}\left(t^{\prime}\right) d t^{\prime}
$$

for $v_{0} \in L^{2}$ and where $W\left(z_{0}, z\right)=W(z)-W\left(z_{0}\right)$. By Ito's formula, the function $v(z, t)=X\left(z_{0}, z\right) v_{0}(t)$ is a.s. the unique solution of the linear equation

$$
v(z, t)=v_{0}(t)+i \sigma_{0} \int_{z_{0}}^{z} \frac{\partial^{2} v}{\partial t^{2}}\left(z^{\prime}, t\right) \circ d W\left(z^{\prime}\right) .
$$

Note that we can get the same solution if we use the general framework introduced in Section 3 for each sample paths of $W$. We can also write the Fourier transform of $X\left(z_{0}, z\right) v_{0}$ as

$$
\mathcal{F}\left(X\left(z_{0}, z\right) v_{0}\right)(\theta)=\mathcal{F}\left(v_{0}\right)(\theta) \exp \left(-i \theta^{2} \sigma_{0} W\left(z_{0}, z\right)\right) .
$$

So, $X\left(z_{0}, z\right): L^{2} \rightarrow L^{2}$ is well-defined. Moreover, by Parseval's theorem, we establish the equality $\left\|X\left(z_{0}, z\right) v_{0}\right\|_{L^{2}}=\left\|v_{0}\right\|_{L^{2}}$.

Following Section 2, there exists a unique solution (that we denote by $u$ ) in $\mathcal{C}\left([0,1], L^{2}\right)$ to the problem $(5.1)$.

One of the main theorems of this section is the following.

TheOREM 5.1. Let $u_{0} \in H^{2}$. If $\sigma_{0}^{2}=2 \int_{0}^{\infty} \mathbb{E}[m(0) m(z)] d z$ then $u^{\varepsilon}$ converges to $u$ in distribution in $\mathcal{C}\left([0,1], L^{2}\right)$ as $\varepsilon$ goes to 0 .

Proof. We define $M^{\varepsilon}(z)=\varepsilon \int_{0}^{z / \varepsilon^{2}} m\left(z^{\prime}\right) d z^{\prime}$ for every $z \in[0,1]$. By classical diffusion-approximation theorems [12], $M^{\varepsilon}$ converges in the space of continuous functions to $\sigma_{0} W$ when $\varepsilon \rightarrow 0$. Hence, by Theorem 3.7, we get the result.

5.2. Diffusion-approximation for the numerical solution. In this subsection we construct an approximation $u^{h}$ for $u$. As for $u^{\varepsilon, h}$, we subdivide $[0,1]$ in $N$ small steps. We let $h=1 / N$. We define: $u_{0}^{h}(t):=u_{0}(t)$ (initial condition), and for every $n \in\{0, \cdots, N-1\}$ :

$$
u_{n}^{h} \rightarrow u_{n+1}^{h}:\left\{\begin{array}{l}
u_{n+1 / 2}^{h}:=X(n h,(n+1) h) u_{n}^{h}, \\
u_{n+1}^{h}:=Y(h) u_{n+1 / 2}^{h}
\end{array}\right.
$$


Finally, $u^{h}$ is defined as: for every $z \in[0,1]$

$$
u^{h}(z)=\sum_{j=0}^{N-1} \mathbb{I}_{z \in[j h,(j+1) h]}\left(u_{j}^{h}+(z / h-[z / h])\left(u_{j+1}^{h}-u_{j}^{h}\right)\right) .
$$

We establish for the limit process analogous error estimates with Theorem 4.1 and 4.6 .

THEOREM 5.2. We assume that $u_{0} \in H^{2}$. There exists a constant $C$ depending only on $g$ and $\left\|u_{0}\right\|_{H^{2}}$, such that for every $h \in(0,1]$, and $n$ satisfying $n h \leq 1$ :

$$
\mathbb{E}\left[\left\|u_{n}^{h}-u(n h, \cdot)\right\|_{L^{2}}^{2}\right] \leq C h .
$$

Moreover, for every $z \in[0,1]$ :

$$
\mathbb{E}\left[\left\|u^{h}(z)-u(z, \cdot)\right\|_{L^{2}}^{2}\right] \leq C h .
$$

Theorem 5.3. Let $u_{0} \in H^{2}$. There exists $C$ such that for every $h \in(0,1]$ and $\gamma>0$ :

$$
\mathbb{P}\left[\sup _{z \in[0,1]}\left\|u^{h}(z)-u(z)\right\|_{L^{2}}>\gamma\right] \leq \frac{C}{\gamma^{4}} h .
$$

The proofs for these theorems are the same as for Theorems 4.1 and 4.6. The main difference is the substitution of Lemma 4.3 by Lemma 5.4 which follows.

Lemma 5.4. Let $W$ be a Brownian motion. For every $l \in \mathbb{N}^{*}$, there exists $C_{l}$ such that

$$
\mathbb{E}\left[\left(\sup _{z^{\prime} \in\left[z_{0}, z\right]}\left|W\left(z^{\prime}\right)-W\left(z_{0}\right)\right|\right)^{2 l}\right] \leq C_{l}\left(z-z_{0}\right)^{l} .
$$

The proof of Lemma 5.4 is a direct consequence of Burkholder's theorem [10].

We establish the convergence of $u^{\varepsilon, h}$ to $u^{h}$ as $\varepsilon$ goes to 0 .

Theorem 5.5. Let $u_{0} \in L^{2}$ and $N \in \mathbb{N}$. We fix $h=1 / N$. Then $u^{\varepsilon, h}$ converges in distribution in $\mathcal{C}\left([0,1], L^{2}\right)$ to $u^{h}$ as $\varepsilon \rightarrow 0$.

Proof. We begin the proof by showing that for a fixed $N$, the sequence $\left(u_{0}, u_{1}^{\varepsilon, h}, \cdots, u_{N}^{\varepsilon, h}\right)$ converges to $\left(u_{0}, u_{1}^{h}, \cdots, u_{N}^{h}\right)$ in distribution in $\left(L^{2}\right)^{N+1}$ as $\varepsilon$ goes to 0 . It is sufficient to show that for every $n \leq N$ the sequence $\left(M^{\varepsilon}, u_{0}, u_{1 / 2}^{\varepsilon, h}, u_{1}^{\varepsilon, h}, \cdots, u_{n-1 / 2}^{\varepsilon, h}, u_{n}^{\varepsilon, h}\right)$ converges to $\left(\sigma_{0} W, u_{0}, u_{1 / 2}^{h}, u_{1}^{h}, \cdots, u_{n-1 / 2}^{h}, u_{n}^{h}\right)$ in distribution in $\mathcal{C}([0,1], \mathbb{R}) \times\left(L^{2}(\mathbb{R}, \mathbb{C})\right)^{2 n+1}$ as $\varepsilon \rightarrow 0$, where $M^{\varepsilon}(z)=\varepsilon \int_{0}^{z / \varepsilon^{2}} m\left(z^{\prime}\right) d z^{\prime}$ for every $z \in[0,1]$. We proceed recursively on $n$.

Let $n=0$. By diffusion-approximation theorems [12], $M^{\varepsilon}$ converges in distribution in $\mathcal{C}([0,1], \mathbb{R})$ to $\sigma_{0} W$ as $\varepsilon$ goes to $0 . u_{0}$ is not random, then $\left(M^{\varepsilon}, u_{0}\right)$ converges in distribution in $\mathcal{C}([0,1], \mathbb{R}) \times L^{2}$ to $\left(\sigma_{0} W, u_{0}\right)$.

Now we assume the convergence of $\left(M^{\varepsilon}, u_{0}, u_{1 / 2}^{\varepsilon, h}, u_{1}^{\varepsilon, h}, \cdots, u_{n-1 / 2}^{\varepsilon, h}, u_{n}^{\varepsilon, h}\right)$ and we prove the convergence of $\left(M^{\varepsilon}, u_{0}, u_{1 / 2}^{\varepsilon, h}, u_{1}^{\varepsilon, h}, \cdots, u_{n+1 / 2}^{\varepsilon, h}, u_{n+1}^{\varepsilon, h}\right)$. The Fourier transform of $u_{n+1 / 2}^{\varepsilon, h}$ is written as

$$
\widehat{u}_{n+1 / 2}^{\varepsilon, h}(\theta)=\widehat{u}_{n}^{\varepsilon, h} \exp \left(-i \theta^{2}\left(M^{\varepsilon}((n+1) h)-M^{\varepsilon}(n h)\right)\right) .
$$


Let a sequence $\left(\left(w_{k}, v_{k}\right)\right)_{k}$ which converge in $\mathcal{C}([0,1], \mathbb{R}) \times L^{2}$ to a limit $(w, v)$. We have

$$
\begin{aligned}
& \left\|v_{k} \exp \left(-i \theta^{2}\left(w_{k}((n+1) h)-w_{k}(n h)\right)\right)-v \exp \left(-i \theta^{2}(w((n+1) h)-w(n h))\right)\right\|_{L^{2}} \\
& \leq\left\|v_{k}-v\right\|_{L^{2}}+\left\|v-v \exp \left(-i \theta^{2}\left(w((n+1) h)-w(n h)-w_{k}((n+1) h)+w_{k}(n h)\right)\right)\right\|_{L^{2}} .
\end{aligned}
$$

The last term of the inequality goes to 0 by the bounded convergence theorem, hence, $u_{n+1 / 2}^{\varepsilon, h}$ is written as a continuous function of $\left(M^{\varepsilon}, u_{0}, u_{1 / 2}^{\varepsilon, h}, u_{1}^{\varepsilon, h}, \cdots, u_{n-1 / 2}^{\varepsilon, h}, u_{n}^{\varepsilon, h}\right)$. So the convergence in distribution of $\left(M^{\varepsilon}, u_{0}, u_{1 / 2}^{\varepsilon, h}, u_{1}^{\varepsilon, h}, \cdots, u_{n}^{\varepsilon, h}, u_{n+1 / 2}^{\varepsilon, h}\right)$ to $\left(\sigma_{0} W, u_{0}, u_{1 / 2}^{h}, u_{1}^{h}, \cdots, u_{n}^{h}, u_{n+1 / 2}^{h}\right)$ as $\varepsilon$ goes to 0 holds. Now we deal with $\left(M^{\varepsilon}, u_{0}, u_{1 / 2}^{\varepsilon, h}, u_{1}^{\varepsilon, h}, \cdots, u_{n+1 / 2}^{\varepsilon, h}, u_{n+1}^{\varepsilon, h}\right)$. We have

$$
u_{n+1}^{\varepsilon, h}=u_{n+1 / 2}^{\varepsilon, h} \exp \left(i h f\left(\left|u_{n+1 / 2}^{\varepsilon, h}\right|^{2}\right)\right)=Y(h) u_{n+1 / 2}^{\varepsilon, h} .
$$

By Lemma $3.9 Y(h)$ is a Lipschitz map from $L^{2}$ to $L^{2}$. So $u_{n+1}^{\varepsilon, h}$ can be written as a continuous function of $\left(M^{\varepsilon}, u_{0}, u_{1 / 2}^{\varepsilon, h}, u_{1}^{\varepsilon, h}, \cdots, u_{n+1 / 2}^{\varepsilon, h}\right)$, hence the convergence of $\left(M^{\varepsilon}, u_{0}, u_{1 / 2}^{\varepsilon, h}, u_{1}^{\varepsilon, h}, \cdots, u_{n+1 / 2}^{\varepsilon, h}, u_{n+1}^{\varepsilon, h}\right)$ to $\left(\sigma_{0} W, u_{0}, u_{1 / 2}^{h}, u_{1}^{h} \cdots, u_{n+1 / 2}^{h}, u_{n+1}^{h}\right)$ as $\varepsilon$ goes to 0 holds.

Hence, $\quad\left(M^{\varepsilon}, u_{0}, u_{1 / 2}^{\varepsilon, h}, u_{1}^{\varepsilon, h}, \cdots, u_{N-1 / 2}^{\varepsilon, h}, u_{N}^{\varepsilon, h}\right) \quad$ converges $\quad$ in distribution in $\mathcal{C}([0,1], \mathbb{R}) \times\left(L^{2}\right)^{2 N+1} \quad$ to $\left(\sigma_{0} W, u_{0}, u_{1 / 2}^{h}, u_{1}^{h}, \cdots, u_{N-1 / 2}^{h}, u_{N}^{h}\right) \quad$ as $\quad \varepsilon \rightarrow 0 . \quad$ Hence, $\left(u_{0}, u_{1}^{\varepsilon, h}, \cdots, u_{N}^{\varepsilon, h}\right)$ converges in distribution in $\left(L^{2}\right)^{N+1}$ as $\varepsilon$ goes to 0 to $\left(u_{0}, u_{1}^{h}, \cdots, u_{N}^{h}\right)$. To conclude it remains to prove the continuity of the map

$$
\begin{aligned}
\Psi:\left(L^{2}\right)^{N+1} & \rightarrow \mathcal{C}\left([0,1], L^{2}\right) \\
\left(v_{0}, \cdots, v_{N}\right) & \mapsto z \mapsto \sum_{j=0}^{N-1} \mathbb{I}_{z \in[j h,(j+1) h]}\left(v_{j}+(z / h-[z / h])\left(v_{j+1}-v_{j}\right)\right) .
\end{aligned}
$$

Let $\left(v_{0}, \cdots, v_{N}\right)$ and $\left(\widetilde{v}_{0}, \cdots, \widetilde{v}_{N}\right)$ in $\left(L^{2}\right)^{N+1}$. If $z \in[j h,(j+1) h]$, then

$$
\left\|\Psi\left(v_{0}, \cdots, v_{N}\right)(z)-\Psi\left(\widetilde{v}_{0}, \cdots, \widetilde{v}_{N}\right)(z)\right\|_{L^{2}} \leq 2\left\|v_{j}-\widetilde{v}_{j}\right\|_{L^{2}}+\left\|v_{j+1}-\widetilde{v}_{j+1}\right\|_{L^{2}} .
$$

Hence,

$$
\begin{aligned}
\sup _{z \in[0,1]}\left\|\Psi\left(v_{0}, \cdots, v_{N}\right)(z)-\Psi\left(\widetilde{v}_{0}, \cdots, \widetilde{v}_{N}\right)(z)\right\|_{L^{2}} \leq & 2 \sum_{j=0}^{N-1}\left\|v_{j}-\widetilde{v}_{j}\right\|_{L^{2}} \\
& +\sum_{j=0}^{N-1}\left\|v_{j+1}-\widetilde{v}_{j+1}\right\|_{L^{2}} .
\end{aligned}
$$

This concludes the proof.

5.3. Link between diffusion-approximation and splitting scheme. In this subsection we show that the robustness of the splitting scheme allows us to propose a new proof for Theorem 5.1. This can be illustrated by the following commutative diagram:

$$
\begin{aligned}
& u^{\varepsilon, h} \stackrel{\varepsilon \rightarrow 0}{\longrightarrow} u^{h} \\
& \downarrow h \rightarrow 0 \quad \downarrow h \rightarrow 0 \\
& u^{\varepsilon} \stackrel{\varepsilon \rightarrow 0}{\longrightarrow} u .
\end{aligned}
$$


Proof. (Theorem 5.1) By Portmanteau's theorem [6], we show that for every bounded Lipschitz function $\Phi: \mathcal{C}\left([0,1], L^{2}\right) \rightarrow \mathbb{R}$ we have

$$
\lim _{\varepsilon \rightarrow 0} \mathbb{E}\left[\Phi\left(u^{\varepsilon}\right)\right]=\mathbb{E}[\Phi(u)]
$$

We let $\Delta^{\varepsilon}=\left|\mathbb{E}\left[\Phi\left(u^{\varepsilon}\right)\right]-\mathbb{E}[\Phi(u)]\right|$ and $\|v\|:=\sup _{z \in[0,1]}\|v(z, \cdot)\|_{L^{2}}$ for $v \in \mathcal{C}\left([0,1], L^{2}\right)$. Let $M>0$ and $\delta>0$. By Markov's inequality and Theorems 4.6 and 5.3 we have:

$$
\begin{aligned}
0 \leq \Delta^{\varepsilon} \leq & \left|\mathbb{E}\left[\Phi\left(u^{\varepsilon}\right)-\Phi\left(u^{\varepsilon, h}\right)\right]\right|+\left|\mathbb{E}\left[\Phi\left(u^{h}\right)\right]-\mathbb{E}\left[\Phi\left(u^{\varepsilon, h}\right)\right]\right|+\left|\mathbb{E}\left[\Phi\left(u^{h}\right)-\Phi(u)\right]\right| \\
\leq & \left|\mathbb{E}\left[\Phi\left(u^{h}\right)\right]-\mathbb{E}\left[\Phi\left(u^{\varepsilon, h}\right)\right]\right|+2 \sup _{\left\{\|x\| \leq M,\left\|x-x^{\prime}\right\| \leq \delta\right\}}\left|\Phi(x)-\Phi\left(x^{\prime}\right)\right| \\
& +2\|\Phi\|_{\infty}\left(2 \frac{\mathbb{E}\left[\left\|u_{0}\right\|^{2}\right]}{M^{2}}+2 \frac{h+\varepsilon^{4} / h}{\delta^{4}}\right) .
\end{aligned}
$$

So by Theorem (5.5), for every $M, \delta$ and $h$, we have

$$
\limsup _{\varepsilon \rightarrow 0} \Delta^{\varepsilon} \leq 2 \sup _{\left\{\|x\| \leq M,\left\|x-x^{\prime}\right\| \leq \delta\right\}}\left|\Phi(x)-\Phi\left(x^{\prime}\right)\right|+4\|\Phi\|_{\infty}\left(\frac{\mathbb{E}\left[\left\|u_{0}\right\|^{2}\right]}{M^{2}}+\frac{h}{\delta^{4}}\right) .
$$

First we let $h \rightarrow 0$, then $\delta \rightarrow 0$ and finally $M \rightarrow \infty$ to complete the proof.

\section{Cubic nonlinear Schrödinger equation}

In this section we deal with the cubic nonlinear Schrödinger equation which is a more realistic model for pulse propagation in an optical fiber:

$$
i \frac{\partial u^{\varepsilon}}{\partial z}+\frac{1}{\varepsilon} m\left(\frac{z}{\varepsilon^{2}}\right) \frac{\partial^{2} u^{\varepsilon}}{\partial t^{2}}+\left|u^{\varepsilon}\right|^{2} u^{\varepsilon}=0
$$

We introduce some notations. For every $M>0$, we define the truncation function $f_{M} \in \mathcal{C}^{\infty}\left(\mathbb{R}_{+}, \mathbb{R}_{+}\right)$by $f_{M}(x)=x$ if $x \leq M, f_{M}(x)=3 M / 2$ if $x \geq 2 M,\left\|f_{M}^{\prime}\right\|_{\infty} \leq 1$ and $\left\|f_{M}^{\prime \prime}\right\|_{\infty} \leq 1$. So, the complex function $g_{M}: \zeta \mapsto f_{M}\left(|\zeta|^{2}\right) \zeta$ and all its partial derivatives are Lipschitz. For a fixed $M$, we introduce:

$$
i \frac{\partial u^{\varepsilon, M}}{\partial z}+\frac{1}{\varepsilon} m\left(\frac{z}{\varepsilon^{2}}\right) \frac{\partial^{2} u^{\varepsilon, M}}{\partial t^{2}}+f_{M}\left(\left|u^{\varepsilon, M}\right|^{2}\right) u^{\varepsilon, M}=0 .
$$

The main result of this section is the following.

Lemma 6.1. Let $u_{0} \in H^{2}$. Then, there exists $Z_{0}>0$ and $M_{0}>0$ depending only on $\left\|u_{0}\right\|_{H^{1}}$ such that for every $\varepsilon \in(0,1), z \in\left[0, Z_{0}\right]$ and $M>M_{0}, u^{\varepsilon, M}(z, \cdot)=u^{\varepsilon, M_{0}}(z, \cdot)$, $\left\|u^{\varepsilon, M}(z, \cdot)\right\|_{\infty}^{2} \leq M_{0}$, and $u^{\varepsilon, M_{0}}$ is the unique solution of $(6.1)$ on $\left[0, Z_{0}\right]$.

Before proving Lemma 6.1, we establish a technical lemma.

Lemma 6.2. Let $\phi:[0,1] \rightarrow \mathbb{R}$ a nonnegative continuous function and $C$ a constant such that for every $z \in[0,1]$ :

$$
\phi(z) \leq \phi(0)+C \int_{0}^{z} \phi\left(z^{\prime}\right)^{3} d z^{\prime}
$$

Then for every $z \in\left[0,\left(2 C \phi(0)^{2}\right)^{-1}\right)$,

$$
\phi(z) \leq \frac{1}{\sqrt{\phi(0)^{-2}-2 C z}} .
$$


Note that this estimate holds only for finite $z$ but is sufficient in our framework.

Proof. We assume $\phi$ to be differentiable. We have $\phi^{\prime}(z) / \phi(z)^{3} \leq C$, then $\int_{0}^{z} \phi^{\prime}\left(z^{\prime}\right) / \phi\left(z^{\prime}\right)^{3} d z^{\prime} \leq C z$, and for every $z \in\left[0,\left(2 C \phi(0)^{2}\right)^{-1}\right)$,

$$
\phi(z) \leq \frac{1}{\sqrt{\phi(0)^{-2}-2 C z}} .
$$

We extend this result to every continuous functions by density.

Proof. (Lemma 6.1) By an explicit computation, we verify $\left\|u^{\varepsilon, M}\right\|_{L^{2}}=\left\|u_{0}\right\|_{L^{2}}$. Moreover we have

$$
u^{\varepsilon, M}(z, t)=X^{\varepsilon}(0, z) u_{0}(t)+i \int_{0}^{z} X^{\varepsilon}\left(z^{\prime}, z\right) g_{M}\left(u^{\varepsilon, M}\left(z^{\prime}, t\right)\right) d z^{\prime}
$$

so

$$
\partial_{t} u^{\varepsilon, M}(z, t)=X^{\varepsilon}(0, z) \partial_{t} u_{0}(t)+i \int_{0}^{z} X^{\varepsilon}\left(z^{\prime}, z\right) \partial_{t} u^{\varepsilon, M}\left(z^{\prime}, t\right) g_{M}^{\prime}\left(u^{\varepsilon, M}\left(z^{\prime}, t\right)\right) d z^{\prime},
$$

then

$$
\begin{aligned}
\left\|\partial_{t} u^{\varepsilon, M}(z)\right\|_{L^{2}} \leq & \left\|\partial_{t} u_{0}\right\|_{L^{2}}+\int_{0}^{z}\left\|\partial_{t} u^{\varepsilon, M}\left(z^{\prime}\right) g_{M}^{\prime}\left(u^{\varepsilon, M}\left(z^{\prime}\right)\right)\right\|_{L^{2}} d z^{\prime} \\
\leq & \left\|\partial_{t} u_{0}\right\|_{L^{2}}+C \int_{0}^{z}\left\|\partial_{t} u^{\varepsilon, M}\left(z^{\prime}\right)\left|u^{\varepsilon, M}\left(z^{\prime}\right)\right|^{2}\right\|_{L^{2}} d z^{\prime} \\
& \left(C \text { independant of } M, \text { only depending on } g \text { and } u_{0}\right) \\
\leq & \left\|\partial_{t} u_{0}\right\|_{L^{2}}+C \int_{0}^{z}\left\|u^{\varepsilon, M}\left(z^{\prime}\right)\right\|_{\infty}^{2}\left\|\partial_{t} u^{\varepsilon, M}\left(z^{\prime}\right)\right\|_{L^{2}} d z^{\prime} \\
\leq & \left\|\partial_{t} u_{0}\right\|_{L^{2}}+C \int_{0}^{z}\left\|u^{\varepsilon, M}\left(z^{\prime}\right)\right\|_{H^{1}}^{3} d z^{\prime}
\end{aligned}
$$

(Sobolev's inequality),

hence

$$
\left\|u^{\varepsilon, M}(z)\right\|_{H^{1}} \leq\left\|u_{0}\right\|_{H^{1}}+C \int_{0}^{z}\left\|u^{\varepsilon, M}\left(z^{\prime}\right)\right\|_{H^{1}}^{3} d z^{\prime} .
$$

Following Lemma 6.2 , for every $z \in\left[0,\left(2 C\left\|u_{0}\right\|_{H^{1}}^{2}\right)^{-1}\right)$,

$$
\left\|u^{\varepsilon, M}(z)\right\|_{H^{1}} \leq \frac{1}{\sqrt{\left\|u_{0}\right\|_{H^{1}}^{-2}-2 C z}} .
$$

This upper bound does not depend on $M$. We choose $Z_{0}<\left(2 C\left\|u_{0}\right\|_{H^{1}}^{2}\right)^{-1}$. For every $z \leq Z_{0}$, we have

$$
\left\|u^{\varepsilon, M}(z)\right\|_{H^{1}}^{2} \leq K_{0}:=\left(\left\|u_{0}\right\|_{H^{1}}^{-2}-2 C Z_{0}\right)^{-1} .
$$

By Sobolev's embedding $H^{1} \subset L^{\infty}$

$$
\left\|u^{\varepsilon, M}(z)\right\|_{\infty}^{2} \leq M_{0}:=C_{s o b} K_{0}
$$


As $f_{M}(x)=x$ for every $x \leq M$, the solution $u^{\varepsilon, M}$ of (6.2) does not depend on $M$ and is also solution of (6.1). This concludes the proof by uniqueness of the solution.

An important consequence of Lemma 6.1 is the extension of result for (4.1) to (6.1). We define as solution of (6.1) with initial condition $u_{0} \in H^{2}$ the solution which is defined in 6.2 for a sufficiently great $M$. Note that the same procedure can be used to define the solution of the equation:

$$
u(z, t)=u_{0}(t)+i \sigma_{0} \int_{0}^{z} \frac{\partial^{2} u}{\partial t^{2}}\left(z^{\prime}, t\right) \circ d W\left(z^{\prime}\right)+i \int_{0}^{z}\left|u\left(z^{\prime}, t\right)\right|^{2} u\left(z^{\prime}, t\right) d z^{\prime} .
$$

Moreover, the error estimate for the pseudo-spectral split-step scheme and the diffusion-approximation theorem can be established.

Theorem 6.3. Let $u_{0} \in H^{2}, u^{\varepsilon}$ (resp. u) the solution of (6.1) (resp. (6.3)) defined as previously on $\left[0, Z_{0}\right]$ where $Z_{0}$ is defined in Lemma $6.1,\left(u^{\varepsilon, h}\right)_{h}$ be the pseudo-spectral split-step scheme for $u^{\varepsilon}$ defined as in Section 4. Then:

(i) there exists $C$ depending only on $M_{0}$ defined in Lemma 6.1 such that for every $\varepsilon<1, h \in(0,1]$, and $n$ satisfying $n h \leq Z_{0}$ :

$$
\mathbb{E}\left[\left\|u_{n}^{\varepsilon, h}-u^{\varepsilon}(n h, \cdot)\right\|_{L^{2}}^{2}\right] \leq C\left(h+\varepsilon^{2}\right) .
$$

(ii) If $\sigma_{0}^{2}=2 \int_{0}^{\infty} \mathbb{E}[m(0) m(z)] d z$, then, $u^{\varepsilon}$ converges in distribution in $\mathcal{C}\left(\left[0, Z_{0}\right], L^{2}\right)$ to $u$.

\section{Optimal error estimate for numerical scheme}

We consider in this section the pseudo-spectral split-step scheme for Equation (5.1) for the effective pulse propagation in an optical fiber. In previous sections, we have shown by operator-theoretic proofs that the splitting method is an approximation of the exact solution at least of order 1 in $h$. In this section we use a formal approach to compute a Taylor's development of the error. So it seems that the splitting method is in fact an approximation of order 2, that is to say:

$$
\mathbb{E}\left[\left\|u_{n}^{h}-u(n h, \cdot)\right\|_{L^{2}}^{2}\right] \leq C h^{2} .
$$

It would be ideal to present a rigorous proof of this estimate at a mathematical level as the one presented previously. However the situation turns out to be tricky because the Taylor's development follows very fastidious calculations. We shall check in Section 8 by numerical simulations.

We recall and introduce some notations. We consider:

$$
\begin{aligned}
& u(z, t)=u_{0}(t)+i \sigma_{0} \int_{z_{0}}^{z} \frac{\partial^{2} u}{\partial t^{2}}\left(z^{\prime}, t\right) \circ d W\left(z^{\prime}\right)+i \int_{z_{0}}^{z} f\left(\left|u\left(z^{\prime}, t\right)\right|^{2}\right) u\left(z^{\prime}, t\right) d z^{\prime}, \\
& v(z, t)=u_{0}(t)+i \sigma_{0} \int_{z_{0}}^{z} \frac{\partial^{2} v}{\partial t^{2}}\left(z^{\prime}, t\right) \circ d W\left(z^{\prime}\right) \\
& w(z, t)=u_{0}(t)+i \int_{z_{0}}^{z} f\left(\left|w\left(z^{\prime}, t\right)\right|^{2}\right) w\left(z^{\prime}, t\right) d z^{\prime} .
\end{aligned}
$$

We denote respectively by $S\left(z_{0}, z\right), X\left(z_{0}, z\right)$ and $Y\left(z-z_{0}\right)$ the flows associated to (7.2), (7.3) and (7.4) with initial conditions on $z_{0}$ and we let $Z\left(z_{0}, z\right):=Y\left(z-z_{0}\right) X\left(z_{0}, z\right)$, $S_{n}^{h}:=S((n-1) h, n h), Z_{n}^{h}:=Z((n-1) h, n h), S_{l \rightarrow n}^{h}:=S_{n}^{h} \cdots S_{l}^{h}$ and $Z_{l \rightarrow n}^{h}:=Z_{n}^{h} \cdots Z_{l}^{h}$, if $l<n$. Here the bracket $\langle\cdot, \cdot\rangle$ (resp. $\langle\cdot, \cdot\rangle_{L^{2}}$ ) stands for the scalar product in $\mathbb{R}^{2}$ (resp. $\left.L^{2}\right)$. 
We begin by the computation of local error. Note that the notation $\mathcal{O}$ is formal. We have

$$
S\left(z_{0}, z\right) u_{0}=X\left(z_{0}, z\right) u_{0}+i \int_{z_{0}}^{z} X\left(z^{\prime}, z\right) g\left(S\left(z_{0}, z^{\prime}\right) u_{0}\right) d z^{\prime}
$$

and

$$
g\left(S\left(z_{0}, z^{\prime}\right) u_{0}\right)=g\left(u_{0}\right)+\left\langle\left(X\left(z_{0}, z^{\prime}\right) u_{0}-u_{0}\right), g^{\prime}\left(u_{0}\right)\right\rangle+\mathcal{O}\left(z^{\prime}-z_{0}\right)
$$

then

$$
\begin{aligned}
S\left(z_{0}, z\right) u_{0}= & X\left(z_{0}, z\right) u_{0}+i\left(z-z_{0}\right) g\left(u_{0}\right) \\
& +i \int_{z_{0}}^{z}\left\langle\left(X\left(z_{0}, z^{\prime}\right) u_{0}-u_{0}\right), g^{\prime}\left(u_{0}\right)\right\rangle d z^{\prime}+\mathcal{O}\left(\left(z-z_{0}\right)^{2}\right) .
\end{aligned}
$$

In the same way we get

$$
\begin{aligned}
Z\left(z_{0}, z\right) u_{0}= & X\left(z_{0}, z\right) u_{0}+i\left(z-z_{0}\right) g\left(u_{0}\right) \\
& +i\left(z-z_{0}\right)\left\langle\left(X\left(z_{0}, z\right) u_{0}-u_{0}\right), g^{\prime}\left(u_{0}\right)\right\rangle+\mathcal{O}\left(\left(z-z_{0}\right)^{2}\right),
\end{aligned}
$$

so

$$
S\left(z_{0}, z\right) u_{0}-Z\left(z_{0}, z\right) u_{0}=F\left(u_{0}\right) \mathcal{D}\left(z_{0}, z\right)+\mathcal{O}\left(\left(z-z_{0}\right)^{2}\right)
$$

where $\mathcal{D}\left(z_{0}, z\right)=\int_{z_{0}}^{z} D\left(z^{\prime}, z\right) d z^{\prime}$ with $D\left(z_{1}, z_{2}\right)=\sigma_{0}\left(W\left(z_{2}\right)-W\left(z_{1}\right)\right)$, and $F\left(u_{0}\right)=$ $\left\langle\left(\partial^{2} u_{0} / \partial t^{2}\right), g^{\prime}\left(u_{0}\right)\right\rangle-\left(\partial^{2} g\left(u_{0}\right) / \partial t^{2}\right)$.

Now we deal with global error. We have

$$
\begin{aligned}
u_{N}^{h}-u(1, \cdot) & =Z_{N}^{h} \cdots Z_{1}^{h} u_{0}-S_{N}^{h} \cdots S_{1}^{h} u_{0} \\
& =\sum_{j=1}^{N}\left\{Z_{n}^{h} \cdots Z_{j}^{h} S_{j-1}^{h} \cdots S_{1}^{h} u_{0}-Z_{n}^{h} \cdots Z_{j+1}^{h} S_{j}^{h} \cdots S_{1}^{h} u_{0}\right\} .
\end{aligned}
$$

Taking $L^{2}-$ norm:

$$
\left\|u_{N}^{h}-u(1, \cdot)\right\|_{L^{2}}^{2} \leq A+B
$$

where

$$
A=\sum_{j=1}^{N}\left\|Z_{n}^{h} \cdots Z_{j}^{h} S_{j-1}^{h} \cdots S_{1}^{h} u_{0}-Z_{n}^{h} \cdots Z_{j+1}^{h} S_{j}^{h} \cdots S_{1}^{h} u_{0}\right\|_{L^{2}}^{2}
$$

and

$$
\begin{aligned}
B= & \sum_{j, k=1, j \neq k}^{N}\left\langle Z_{j \rightarrow n}^{h} S_{1 \rightarrow j-1}^{h} u_{0}-Z_{j+1 \rightarrow n}^{h} S_{1 \rightarrow j}^{h} u_{0},\right. \\
& \left.Z_{k \rightarrow n}^{h} S_{1 \rightarrow k-1}^{h} u_{0}-Z_{k+1 \rightarrow n}^{h} S_{1 \rightarrow k}^{h} u_{0}\right\rangle_{L^{2}},
\end{aligned}
$$

where $S_{l_{0} \rightarrow l_{1}}^{h}$ (resp. $Z_{l_{0} \rightarrow l_{1}}^{h}$ ) denotes $S_{l_{1}}^{h} \cdots S_{l_{0}}^{h}$ (resp. $Z_{l_{1}}^{h} \cdots S_{l_{0}}^{h}$ ). As in the proof of Theorem 4.1 we get $\mathbb{E}[A] \leq C h^{2}$. Now we want to prove a similar inequality for $B$. 
We first consider the rough approximation $Z_{n}^{h} \approx 1$. Letting $\mathcal{D}_{j}^{h}:=\mathcal{D}((j-1) h, j h)$ and using (7.5) we have

$$
\mathbb{E}[B] \approx\left(\sum_{j, k=1, j<k}^{N}+\sum_{j, k=1, j>k}^{N}\right) \mathbb{E}\left[\mathcal{D}_{j}^{h} \mathcal{D}_{k}^{h}\left\langle F\left(S_{1 \rightarrow j-1}^{h} u_{0}\right), F\left(S_{1 \rightarrow k-1}^{h} u_{0}\right)\right\rangle_{L^{2}}\right] .
$$

So, by independence of the increments of the Brownian motion, if $j>k$ we can write

$$
\mathbb{E}\left[\mathcal{D}_{j}^{h}\right] \mathbb{E}\left[\mathcal{D}_{k}^{h}\left\langle F\left(S_{1 \rightarrow j-1}^{h} u_{0}\right), F\left(S_{1 \rightarrow k-1}^{h} u_{0}\right)\right\rangle_{L^{2}}\right],
$$

but $\mathbb{E}\left[\mathcal{D}_{j}^{h}\right]=0$, then, in a first approximation, $\mathbb{E}[B] \approx 0$.

Now we consider a more precise development for $Z_{n}^{h}$. If we let $D_{n}^{h}:=D((n-$ 1) $h, n h)$, then we have $Z_{n}^{h} u_{0} \approx u_{0}+i D_{n}^{h} \partial^{2} u_{0} / \partial t^{2}$, so

$$
Z_{j \rightarrow n}^{h} u_{0} \approx u_{0}+i \sum_{l=j}^{n} D_{l}^{h} \frac{\partial^{2} u_{0}}{\partial t^{2}} .
$$

Then, by calculations as previously and the fact that $W$ is centered and with independent increments, we get $\mathbb{E}[B] \approx 0$.

We conclude this section by the conjecture: there exist a constant $C$ only depending on $g$ and $u_{0}$ such that

$$
\mathbb{E}\left[\left\|u_{n}^{h}-u(n h, \cdot)\right\|_{L^{2}}^{2}\right] \leq C h^{2} .
$$

\section{Numerical simulations}

In this section we solve

$$
i \frac{\partial u^{\varepsilon}}{\partial z}+\frac{1}{\varepsilon} m\left(\frac{z}{\varepsilon^{2}}\right) \frac{\partial^{2} u^{\varepsilon}}{\partial t^{2}}+2\left|u^{\varepsilon}\right|^{2} u^{\varepsilon}=0 .
$$

We implement the splitting scheme introduced in this paper. The aim of this section is to estimate by numerical methods the error between the numerical solution and the exact solution.

First we simulate the random dispersion coefficient. In previous sections we assume that $m$ is a bounded and Markov process, in fact to simplify presentation of the proofs. All of what precedes can be done in a greater generality. The most important is that $m$ has rapidly decaying correlations. For instance, it can be assumed that $m$ is mixing [11]. For a covariance function $r$, we define the dispersion process $m$ by: $m(z)=\int_{-\infty}^{+\infty} \exp (-i z \zeta) \sqrt{\widehat{r}(\zeta)} W(d \zeta)$, where $\widehat{r}$ is the Fourier transform of $r$ and $W$ is a Gaussian measure. Then $m$ is a Gaussian process which is centered, stationary and its covariance function is $r$. Figure 8.1a give a realization of $m$ when $r(z)=\exp \left(-z^{2} / 2\right)$. Note that such a process is neither bounded, nor a Markov process, but mixing.

To compute the exact solution $u^{\varepsilon}$ of (8.1) for $z=1$ we use the splitting method introduced in this paper. We recall that $X^{\varepsilon}\left(z_{0}, z\right)$ and $Y\left(z-z_{0}\right)$ denotes, respectively, the flows associated to the equations

$$
i \frac{\partial v^{\varepsilon}}{\partial z}+\frac{1}{\varepsilon} m\left(\frac{z}{\varepsilon^{2}}\right) \frac{\partial^{2} v^{\varepsilon}}{\partial t^{2}}=0 \quad \text { and } \quad i \frac{\partial w}{\partial z}+2|w|^{2} w=0,
$$

with initial condition on $z_{0}$. Let $\Delta z$ the elementary length step. We choose a solitontype initial condition:

$$
u_{0}(t)=\frac{1}{\cosh (t)} .
$$


a)

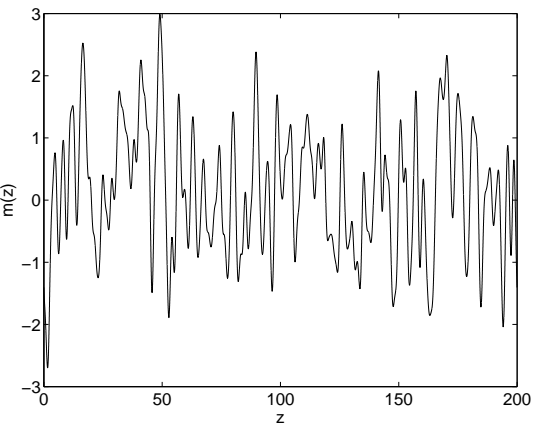

b)

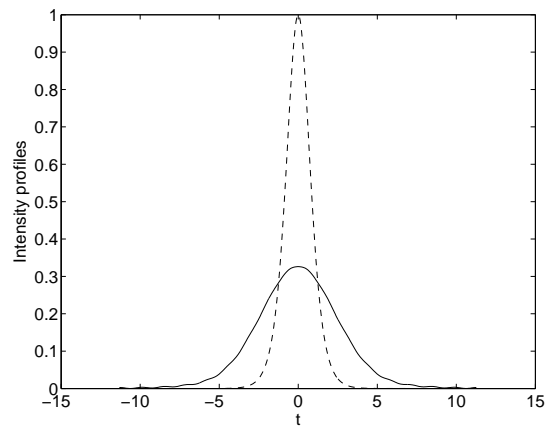

FIG. 8.1. Figure a: A realization of $m$. Figure $b$ : Input intensity profile (dotted lines) output profile (solid line) for $\varepsilon=0.01$.

a)

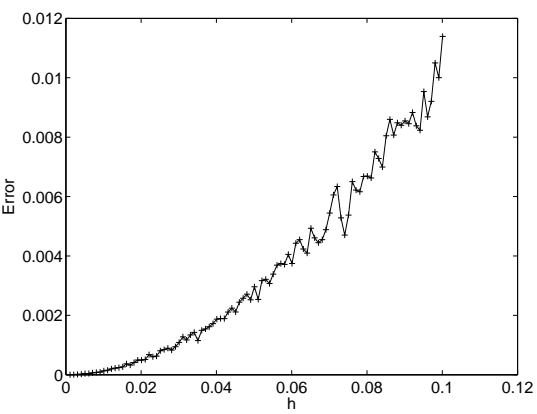

b)

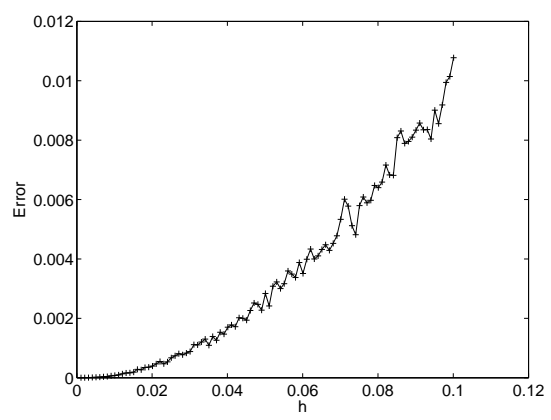

Fig. 8.2. Error $\mathbb{E}\left[\left\|u_{1 / h}^{\varepsilon, h}-u^{\varepsilon}(1, \cdot)\right\|_{L^{2}}^{2}\right]$. Figure a: for $\varepsilon=0.01$. Figure $b$ : for $\varepsilon=0.05$.

For every $n \in\left\{0, \ldots,(\Delta z)^{-1}-1\right\} \quad$ we compute $u_{n+1}^{\varepsilon, \Delta z}(t)=u^{\varepsilon}((n+1) \Delta z, t)$ from $u_{n}^{\varepsilon, \Delta z}(t)=u^{\varepsilon}(n \Delta z, t)$ :

$$
u_{n}^{\varepsilon, \Delta z} \rightarrow u_{n+1}^{\varepsilon, \Delta z}:\left\{\begin{array}{ll}
u_{n+1 / 2}^{\varepsilon, \Delta z}:=X^{\varepsilon}(n \Delta z,(n+1) \Delta z) u_{n}^{\varepsilon, \Delta z} & \text { (First step) } \\
u_{n+1}^{\varepsilon, \Delta z}:=Y(\Delta z) u_{n+1 / 2}^{\varepsilon, \Delta z} & \text { (Second step) }
\end{array} .\right.
$$

In Fourier space the effect of the operator $X^{\varepsilon}(n \Delta z,(n+1) \Delta z)$ is a scalar multiplication by $\exp \left(-(i \theta / \varepsilon) \int_{n \Delta z}^{(n+1) \Delta z} m\left(z^{\prime} / \varepsilon^{2}\right) d z^{\prime}\right)$. Hence, the first step is solved using a Fourier transform:

$$
\begin{aligned}
u_{n}^{\varepsilon, \Delta z}(t) & \stackrel{\mathcal{F}}{\longrightarrow} \\
\downarrow & \widehat{u}_{n}^{\varepsilon, \Delta z}(\theta) \\
\downarrow & \downarrow \\
u_{n+1 / 2}^{\varepsilon, \Delta z}(t) & \stackrel{\mathcal{F}^{-1}}{\longleftarrow} \widehat{u}_{n+1 / 2}^{\varepsilon, \Delta z}(\theta)=\widehat{u}_{n}^{\varepsilon, \Delta z}(\theta) \times \exp \left(-\left(i \theta^{2} / \varepsilon\right) \int_{n \Delta z}^{(n+1) \Delta z} m\left(z^{\prime} / \varepsilon^{2}\right) d z^{\prime}\right) .
\end{aligned}
$$

The second step is solved explicitly by exponentiation:

$$
u_{n+1}^{\varepsilon, \Delta z}=u_{n+1 / 2}^{\varepsilon, \Delta z} \times \exp \left(2 i \Delta z\left|u_{n+1 / 2}^{\varepsilon, \Delta z}\right|^{2}\right) .
$$

Figure $8.1 \mathrm{~b}$ give the input intensity profile and the output profile for a numerical experiment. 
a)

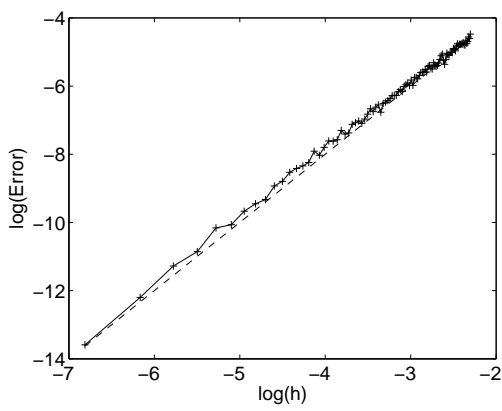

b)

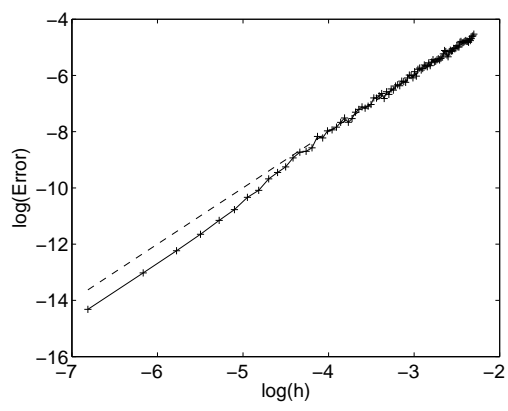

FIG. 8.3. Error $\mathbb{E}\left[\left\|u_{1 / h}^{\varepsilon, h}-u^{\varepsilon}(1, \cdot)\right\|_{L^{2}}^{2}\right]$ in log-log plot (solid line) and the line $y=2 x$ (dashed line). Figure a: for $\varepsilon=0.01$. Figure $b$ : for $\varepsilon=0.05$.

Now we simulate the error $\mathbb{E}\left[\| u_{1 / h}^{\varepsilon, h}-\left.u^{\varepsilon}(1, \cdot)\right|_{L^{2}} ^{2}\right]$ at $z=1$ with respect to $h$. Figures (8.2a) and (8.2b) give the error $\mathbb{E}\left[\left\|u_{1 / h}^{\varepsilon, h}-u^{\varepsilon}(1, \cdot)\right\|_{L^{2}}^{2}\right]$ at $z=1$ with $\varepsilon=0.01$ and $\varepsilon=$ 0.05. Figures (8.3a) and (8.3b) give the error $\mathbb{E}\left[\left\|u_{1 / h}^{\varepsilon, h}-u^{\varepsilon}(1, \cdot)\right\|_{L^{2}}^{2}\right]$ in a log-log plot.

Note that this simulations are in good agreement with formal theoretical result of the previous section: the error is proportional to $h^{2}$.

Acknowledgement. I thank Josselin Garnier for very helpful suggestions on this work.

\section{REFERENCES}

1] G. P. Agrawal, Nonlinear Fiber Optics, 3rd ed., Academic Press, San Diego, 2001.

[2] G. P. Agrawal, Applications of Nonlinear Fiber Optics, Academic Press, San Diego, 2001.

[3] G. Bal and L. Ryzhik, Time splitting for wave equations in random media, M2AN Math. Model. Numer. Anal., 38(6), 961-988, 2004.

[4] G. Bal and L. Ryzhik, Time splitting for the Liouville equation in a random medium, Comm. Math. Sci., 2(3), 515-534, 2004.

[5] C. Besse, B. Bidégaray and S. Descombes, Order estimates in time of splitting methods for the nonlinear Schrödinger equation, SIAM J. Numer. Anal., 40(1), 26-40, 2002.

[6] P. Billingsley, Convergence of Probability Measures, Wiley, 1968.

[7] A. De Bouard and A. Debussche, A stochastic nonlinear Schrödinger equation with multiplicative noise, Comm. Math. Phys., 205, 161-181, 1999.

[8] A. De Bouard, A. Debussche and L. Di Menza, Theoretical and numerical aspects of stochastic nonlinear Schrödinger equations, Monte-Carlo Meth. and Appl., 7(1-2), 55-63, 2001.

[9] P. Donnat, Quelques contributions mathématiques à l'optique non-linéaire, PhD Thesis, école Polytechnique, 1993.

[10] S. N. Ethier and T. G. Kurtz, Markov Processes, Characterization and Convergence, Wiley, New York, 1986.

[11] H. J. Kushner, Approximation and Weak Convergence Methods for Random Processes, MIT Press, Cambridge, 1994

[12] G. Papanicolaou and W. Kohler, Asymptotic theory of mixing stochastic ordinary differential equations, Comm. Pure Appl. Math., 27, 641-668, 1974

[13] G. Papanicolaou, D. W. Stroock and S. R. S. Varadhan, Martingale approach to some limit theorem, in Statistical Mechanics and Dynamical systems, D. Ruelle, ed., Duke Turbulence Conf., Duke Univ. Math. Series III, Part IV, 1-120, 1976.

[14] G. Strang, On the construction and comparison of difference schemes, SIAM J. Numer. Anal. 5, 506-517, 1968. 